\title{
Col-OSSOS: The Colors of the Outer Solar System Origins Survey
}

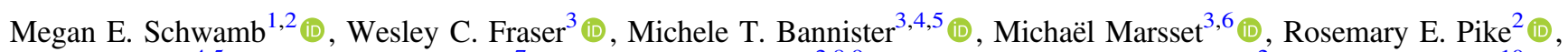 \\ J. J. Kavelaars ${ }^{4,5}$ (1) , Susan D. Benecchi ${ }^{7}$ (1) , Matthew J. Lehner ${ }^{2,8,9}$ (1), Shiang-Yu Wang (王祥宇) $)^{2}$, Audrey Thirouin ${ }^{10} \mathbb{( B D}^{\circ}$,

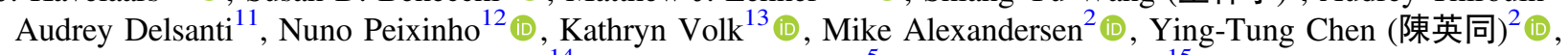 \\ Brett Gladman $^{14}$, Stephen D. J. Gwyn ${ }^{5}$, and Jean-Marc Petit ${ }^{15}$ (iD \\ ${ }^{1}$ Gemini Observatory, Northern Operations Center, 670 North A'ohoku Place, Hilo, HI 96720, USA; mschwamb.astro@gmail.com \\ ${ }^{2}$ Institute of Astronomy and Astrophysics, Academia Sinica; 11F of AS/NTU Astronomy-Mathematics Building, Nr. 1 Roosevelt Rd., Sec. 4, Taipei 10617, Taiwan \\ ${ }^{3}$ Astrophysics Research Centre, Queen's University Belfast, Belfast BT7 1NN, UK \\ ${ }^{4}$ Department of Physics and Astronomy, University of Victoria, Elliott Building, 3800 Finnerty Rd., Victoria, BC V8P 5C2, Canada \\ ${ }^{5}$ Herzberg Astronomy and Astrophysics Research Centre, National Research Council of Canada, 5071 West Saanich Rd., Victoria, BC V9E 2E7, Canada \\ ${ }^{6}$ Department of Earth, Atmospheric and Planetary Sciences, MIT, 77 Massachusetts Ave., Cambridge, MA 02139, USA \\ ${ }^{7}$ Planetary Science Institute, 1700 East Fort Lowell, Suite 106, Tucson, AZ 85719, USA \\ ${ }^{8}$ Department of Physics and Astronomy, University of Pennsylvania, 209 S. 33rd St., Philadelphia, PA 19104, USA \\ ${ }^{9}$ Harvard-Smithsonian Center for Astrophysics, 60 Garden St., Cambridge, MA 02138, USA \\ ${ }^{10}$ Lowell Observatory, 1400 W. Mars Hill Rd., Flagstaff, AZ 86001, USA \\ ${ }^{11}$ Aix Marseille Université, CNRS, LAM (Laboratoire d'Astrophysique de Marseille) UMR 7326, F-13388, Marseille, France \\ ${ }^{12}$ CITEUC - Centre for Earth and Space Science Research of the University of Coimbra, Geophysical and Astronomical Observatory of the University of Coimbra, \\ 3030-004 Coimbra, Portugal \\ ${ }^{13}$ Lunar and Planetary Laboratory, University of Arizona, 1629 E. University Blvd., Tucson, AZ 85721, USA \\ ${ }^{14}$ Department of Physics and Astronomy, University of British Columbia, Vancouver, BC, Canada \\ ${ }^{15}$ Institut UTINAM UMR6213, CNRS, Univ. Bourgogne Franche-Comté, OSU Theta F-25000 Besançon, France \\ Received 2018 September 8; revised 2019 April 2; accepted 2019 April 11; published 2019 July 11
}

\begin{abstract}
The Colours of the Outer Solar System Origins Survey is acquiring near-simultaneous $g, r$, and $J$ photometry of unprecedented precision with the Gemini North Telescope, targeting nearly 100 trans-Neptunian objects (TNOs) brighter than $m_{r}=23.6 \mathrm{mag}$ discovered in the Outer Solar System Origins Survey. Combining the optical and near-infrared photometry with the well-characterized detection efficiency of the Colours of the Outer Solar System Origins Survey target sample will provide the first flux-limited compositional dynamical map of the outer solar system. In this paper, we describe our observing strategy and detail the data reduction processes we employ, including techniques to mitigate the impact of rotational variability. We present optical and near-infrared colors for 35 TNOs. We find two taxonomic groups for the dynamically excited TNOs, the neutral and red classes, which divide at $g-r \simeq 0.75$. Based on simple albedo and orbital distribution assumptions, we find that the neutral class outnumbers the red class, with a ratio of 4:1 and potentially as high as 11:1. Including in our analysis constraints from the cold classical objects, which are known to exhibit unique albedos and $r-z$ colors, we find that within our measurement uncertainty our observations are consistent with the primordial solar system protoplanetesimal disk being neutral class dominated, with two major compositional divisions in grJ color space.
\end{abstract}

Key words: Kuiper belt: general - minor planets, asteroids: general - planets and satellites: dynamical evolution and stability - planets and satellites: formation - surveys

Supporting material: figure set, machine-readable tables

\section{Introduction}

The region beyond Neptune is populated by hundreds of thousands of planetesimals. These trans-Neptunian objects (TNOs) are the fossils left over after the era of planet construction in our solar system. Their orbits are a record of the outer solar system's past evolution (Sections 2.1-2.2), and their surface composition is a window into the conditions in the early planet-forming disk (Sections 2.3-2.4). We developed the Colours of the Outer Solar System Origins Survey (ColOSSOS) to map the surface properties of the trans-Neptunian populations, through near-simultaneous $g_{-}, r$-, and $J$-band observations with the $8.1 \mathrm{~m}$ Frederick C. Gillett Gemini North Telescope.

Col-OSSOS provides color measurements for a set of TNOs that were detected in a flux-limited survey with a wellmeasured detection efficiency. The survey targets TNOs brighter than $m_{r}=23.6 \mathrm{mag}$ found in the Outer Solar System Origins Survey (OSSOS; Bannister et al. 2016, 2018). Col-
OSSOS affords the first opportunity to explore the true frequency of surface colors within the Kuiper Belt, subdivided by dynamical classification. Initial results are discussed in Fraser et al. (2017), Pike et al. (2017), and Marsset et al. (2019). We were also able to compare Col-OSSOS TNO measurements to the first interstellar object, 1I/'Oumuamua (Bannister et al. 2017a).

In this paper we provide an overview of the Col-OSSOS survey, detailing the observing strategy to obtain nearsimultaneous optical and near-infrared (NIR) colors (Section 3) and summarizing our data analysis strategies (Section 4 and Appendices A and B). Our first data release is 35 TNOs (Section 5), which display three color-dynamical taxonomic groups (Section 6.1). We infer the observed and debiased ratio of the two color groups of the red and neutral surfaces within the dynamically excited Kuiper Belt population (Section 6.2). We briefly examine the implications for the radial color distribution in the primordial planetesimal disk from which the excited TNOs originated (Section 6.3). 


\section{The Orbital Structure and Surface Compositions of TNOs}

\subsection{Dynamical Populations}

The main Kuiper Belt (trans-Neptunian region) can be defined as the conglomeration of minor planets on orbits with semimajor axes between 37 and $\sim 50$ au. ${ }^{16}$ The TNO population can be split into two broad dynamical subgroups: the "cold classicals," on near-circular and low-inclination $i \lesssim 5^{\circ}$ orbits, and an overlapping dynamically excited population with $i$ $\gtrsim 5^{\circ}$ (Brown 2001; Bernstein et al. 2004; Fuentes \& Holman 2008; Gladman et al. 2008; Fraser et al. 2010; Petit et al. 2011; Bannister et al. 2018). The cold classicals have very little dynamical excitation, with an inclination width of only $\simeq 2^{\circ}$ (Brown 2001; Kavelaars et al. 2008; Gulbis et al. 2010; Petit et al. 2011). In contrast, the dynamically excited Kuiper Belt has an inclination width of $\sim 14^{\circ}-16^{\circ}$ (Petit et al. 2017).

The dynamically excited population is a highly complex structure with several subclasses. A fifth of the population is locked in mean motion resonances with Neptune (Malhotra 1995; Gladman et al. 2012): their orbits have integer period ratios with Neptune's orbit. Nonresonant TNOs with large eccentricities form the scattering disk, a slowly decaying population. Their semimajor axes evolve owing to active gravitational scattering with Neptune (Gladman et al. 2008; Gomes et al. 2008). The scattering disk feeds into the shortlived Centaur population, which orbits between the giant planets for timescales of tens of megayears (Dones et al. 1999; Tiscareno \& Malhotra 2003), although it may not be the only population contributing to the Centaurs (Yu \& Tremaine 1999; di Sisto et al. 2010; Horner \& Lykawka 2010). In contrast, TNOs on $q \gtrsim 38$ au orbits with large $a$ beyond the $2: 1$ mean motion resonance reside on moderately stable orbits with little or no direct gravitational interactions with Neptune (Emel'yanenko et al. 2003; Gomes et al. 2005b; Gladman et al. 2008; Brasser \& Schwamb 2015). Orbits with $a>250$ au and $q \gtrsim 45$ au are difficult if not impossible for Neptune to directly scatter planetesimals onto during its outward migration (Brasser \& Schwamb 2015); these orbits have unclear origins and may be emplaced by a different dynamical mechanism (Brown et al. 2004; Morbidelli \& Levison 2004; Brasser et al. 2006, 2012; Gladman \& Chan 2006; Kaib \& Quinn 2008; Trujillo \& Sheppard 2014; Batygin \& Brown 2016; Sheppard \& Trujillo 2016; Bannister et al. 2017b; Pfalzner et al. 2018).

\subsection{Migration History and Population Emplacement}

Overwhelming evidence indicates that the Kuiper Belt did not form entirely in situ. Planetesimal-driven giant planet migration that scatters the early solar system's protoplanetesimal disk is the overarching framework (Malhotra 1995; Thommes et al. 1999; Levison et al. 2008; Nesvorný 2015a, 2015b) that best reproduces the observed orbital structure of the trans-Neptunian region. The specifics of the dynamical instability result in different signatures in the Kuiper Belt's present orbital distribution. The dynamically excited population, including most of the resonant objects, was emplaced into the Kuiper Belt through gravitational scattering from inward regions

\footnotetext{
${ }^{16}$ More precisely, between the $\nu 18$ secular resonance and the 2:1 mean motion resonance with Neptune at $47.5 \mathrm{au}$, though the cold classicals extend a few au farther.
}

of the planetesimal disk. Subsequent interactions with Neptune further sculpted the region and are imprinted on the dynamically excited population's present orbital distribution.

The physical properties of the cold classicals - their photometric colors, slope of their size distribution, albedo distribution, and resolved binary fraction-significantly differ from the rest of the Kuiper Belt (e.g., Tegler \& Romanishin 2000; Doressoundiram et al. 2002; Peixinho et al. 2004, 2008; Noll et al. 2008; Brucker et al. 2009; Fraser et al. 2014; Lacerda et al. 2014). Additionally, the widest cold classical binaries would be disrupted if scattered out into the classical belt by Neptune (Parker \& Kavelaars 2010). Together, this evidence suggests that the vast majority of the cold classicals formed in place. Thus, the cold classicals place unique constraints on Neptune's dynamical history, as their orbits must remain largely undisturbed by Neptune's migration as the planet reached its present-day orbit. Recent work by Fraser et al. (2017) using Col-OSSOS measurements shows that this picture is slightly more complicated. Red-colored cold classicals are thought to have originated at their present location, but "blue binaries" (neutral-colored binaries) found within the cold classical belt are thought to be interlopers that formed farther inward and were deposited into the region during the final stages of Neptune migration.

Recent studies have shown that the smoothness of Neptune's migration at late stages of planetesimal-driven migration produces differences in the predicted structure of Kuiper Belt orbits. Nesvorný (2015a, 2015b) showed that Neptune migration with a slow and occasionally jumping "grainy" motion through a cold disk of planetesimals implants objects on sufficiently excited inclinations and eccentricities in the dynamically excited population. Lawler et al. (2018b) find that the best match to the near-resonant distant populations is from grainy migration. Batygin et al. (2011), Ribeiro de Sousa et al. (2018), and Gomes et al. (2018) conclude that a moderately high eccentric phase during Neptune migration is also a viable scenario to reproduce the structure of the cold classical belt.

\subsection{Compositional Surveys}

The past two decades of observations have provided substantial insights into the surface composition of the bright $m_{r}<22$ mag TNOs, which are readily studied via optical and NIR reflectance spectroscopy. Their surfaces are divided into three categories: dwarf planets rich in volatile ices such as methane, ethane, and water ice (Schaller \& Brown 2007; Barkume et al. 2008; Brown 2012), the Haumea collisional family with strong water ice absorption (Brown et al. 2007; Schaller \& Brown 2008; Snodgrass et al. 2010; Trujillo et al. 2011; Carry et al. 2012; Fraser \& Brown 2012), and surfaces devoid of feature-imprinting volatiles other than water ice. The majority of spectroscopically studied TNOs are spectrally featureless. They exhibit a diversity of surfaces, with spectral gradients ranging from solar-neutral colors to redder than solar in optical wavelengths (Alvarez-Candal et al. 2008; Barkume et al. 2008; Barucci et al. 2008, 2011).

For the far more abundant $>22$ mag TNOs, optical and infrared spectroscopy is impossible with current ground- and space-based facilities. We must instead rely on what broadband and narrowband colors reveal by proxy about the optical and NIR spectral slopes. Large surveys of TNO surface colors have used a variety of optical and infrared wavelength measurements to attempt to understand and classify TNO surfaces. 
Doressoundiram et al. (2008), Peixinho et al. (2015), and the MBOSS database ${ }^{17}$ (Hainaut et al. 2012) provide a compilation of surface colors in the published literature.

The first surveys found that TNO surfaces have a broad range of surface colors, from nearly neutral solar colors to very red (Luu \& Jewitt 1996). Some dynamical populations are confined to a color range, or exhibit a bimodality in color. Tegler \& Romanishin (2000) and Doressoundiram et al. (2001) identified that more neutral surfaces exist at higher orbital inclinations; this trend was later identified as largely an effect of the vast majority of cold classical TNOs having red surfaces (Doressoundiram et al. 2001; Tegler et al. 2003). Additional work confirmed the statistical significance of the cold classicals' color distribution as separate from that of other TNOs (Doressoundiram et al. 2001, 2002; Tegler et al. 2003; Doressoundiram et al. 2007; Peixinho et al. 2008). The European Southern Observatory Large Program on Centaurs and TNOs found a continuum of surface colors in the optical and infrared, with relatively linear color slopes from $B$ to $J$ bands (Boehnhardt et al. 2002; Delsanti et al. 2004, 2006; Peixinho et al. 2004). Other photometric surveys identified a bimodality in surface colors of the Centaur population (Peixinho et al. 2003; Tegler \& Romanishin 2003). As the $r>22$ mag TNOs were surveyed, a bimodality in color became apparent in smaller-size TNOs (Fraser \& Brown 2012; Peixinho et al. 2012, 2015; Fraser et al. 2015), with a bimodality in $g-i$ in smaller excited TNOs (Wong \& Brown 2017).

\subsection{Surface Origins in the Protoplanetesimal Disk}

Two distinct models have been put forth to explain the observed diversity of surfaces observed within the small $(<500$ $\mathrm{km}$ in diameter) TNOs. The two proposals suggest different scenarios for how the surface color variation was emplaced in the TNO population before Neptune migration occurred. Each scenario has its own implications for the structure of the early solar system's planetesimal disk. Col-OSSOS aims to distinguish between these two ideas.

In the first model, summarized by Dalle Ore et al. (2013), small TNO surfaces are divided into five discrete types, each with its own unique color, albedo, and composition. The cold classicals fall into their own separate class, while the dynamically excited TNO population is divided into four types. In this scenario, the unique color classes are the direct result of approximately five or more ice lines that existed in the primordial disk: each unique composition corresponds to formation beyond a specific ice line. In the Dalle Ore et al. (2013) model, these compositional classes were caused by a sharply striped primordial disk with five or more compositional boundaries and very little or no cross-mixing between the forming protoplanetesimals. In this scenario, individual compositional classes manifest as groups of objects clustered in different regions of the optical-NIR color space; at least five should be apparent with sufficiently accurate color measurements.

In the second model, of Fraser \& Brown (2012), small TNOs fall into only three compositional classes, which also correlate with dynamical class: the red cold classicals, the neutral dynamically excited objects, and red dynamically excited objects. Fraser \& Brown (2012) suggest that the protoplanetesimal disk did not show a primordial compositional gradient, but rather was compositionally homogenous between 15 and

\footnotetext{
$\overline{17}$ http://www.eso.org/ ohainaut/MBOSS
}

$45 \mathrm{au}$. All planetesimals in this region would have had relatively similar abundances of surface volatiles after formation. The observed compositional classes were thus a result of post-formation evolution, with some objects rapidly losing their light volatile species. What volatile was lost depended only on surface temperature and hence formation location, with each class of TNO predominantly residing at a different distance within the protoplanetesimal disk (Wong \& Brown 2016, 2017). The separate long-term chemical evolution pathways resulted in different surface colors dividing into three broad classes (the red cold classicals, the neutral dynamically excited objects, and the red dynamically excited objects). In the Fraser \& Brown (2012) scenario, the range of surface colors seen in each class is the result of a range of mixing of unique surface materials. Only two taxons will be apparent in the optical and NIR space, each exhibiting a range of optical-NIR colors, with the cold classical objects sharing a taxon with the dynamically excited red objects.

A sample of sufficiently accurate optical and NIR TNO colors should be able to differentiate between the scenarios of Dalle Ore et al. (2013) and Fraser \& Brown (2012) and inform us of the compositional properties of the early planetesimal disk. Combining the dynamics of the ensemble Kuiper Belt together with its physical and chemical properties would create a powerful probe of Neptune's migration and of the compositional structure of the primordial disk from which the TNOs originated. To date, this has proven a challenging task. Most TNO physical property studies examine the hodgepodge set of objects that were discovered by various surveys with different and varying detection biases. Object size and dynamical classification are dependent properties: small TNOs become discoverable when they are near perihelion, at closer heliocentric distances. For example, Centaurs with color measurements are on average much smaller in size than the hot classical objects with measured colors. This has made it difficult, if not impossible, to accurately estimate the true frequency of the different surface color groups in the modernday Kuiper Belt. Thus, a careful sample of TNOs with known discovery biases is necessary to disentangle the effects of observational biases from the color distribution of the intrinsic populations (Pike \& Kavelaars 2013).

\section{Survey Design}

The goal of Col-OSSOS is to produce the first-ever fluxlimited optical and NIR color survey of TNOs, with wellcharacterized and well-quantified biases. We aim to use this sample to test and probe the taxonomic classes within the Kuiper Belt and to produce reliable intrinsic population statistics. The Col-OSSOS program is governed by a set of overarching requirements:

1. A well-understood, flux-limited TNO sample with quantified biases (Section 3.1).

2. Color measurements that can distinguish between the disk models of Dalle Ore et al. (2013) and Fraser \& Brown (2012) (Section 3.2).

3. Homogeneity in our observing scheme (Section 3.3).

4. Observations in different filters acquired as temporally close as possible (Section 3.3).

5. Consistent high photometric quality of the observations for all targets (Section 3.3). 


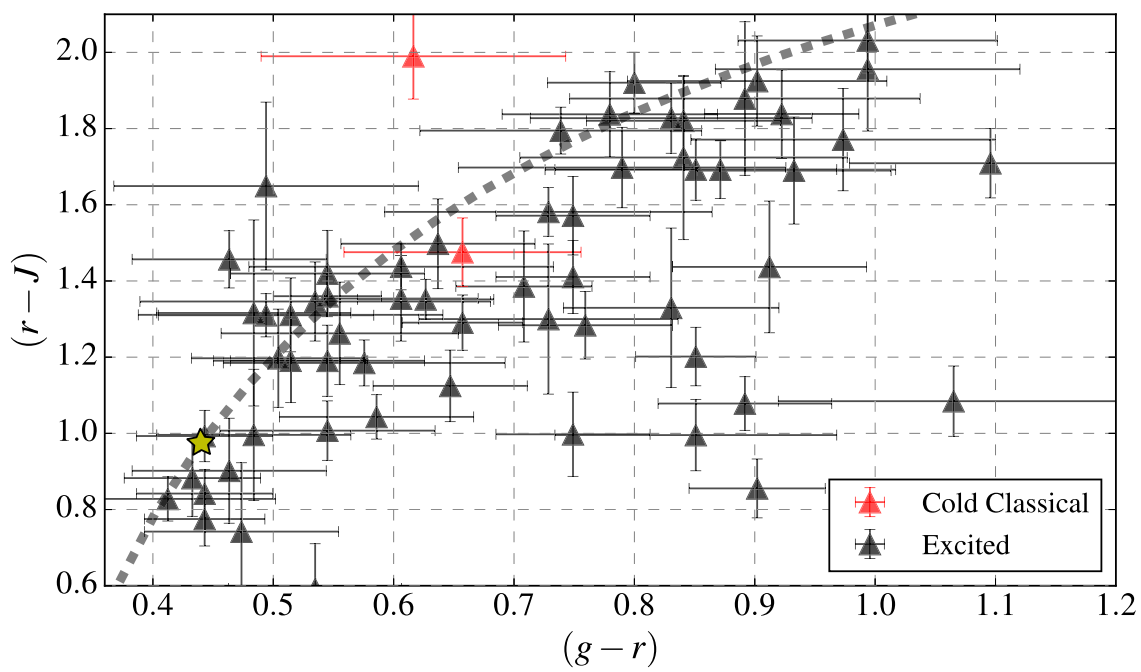

Figure 1. Nonsimultaneous mean observed (converted) $g r J$ color-space measurements of trans-Neptunian populations existing at the conception of the Col-OSSOS survey. Plotted are derived colors for sources with optical and $J$-band photometry, as indexed by MBOSS (Hainaut et al. 2012) or reported by Peixinho et al. (2015). $(g-r)$ was estimated from the reported $(B-V)$ and the conversion of Jester et al. (2005). ( $r-J)$ was estimated by first estimating $(r-i)$ from the reported $(R-I)$ using the conversion reported by Jester et al. (2005) and then estimating $(R-r)$ using the conversion reported by Jordi et al. (2006). ( $R-r)$ is used to estimate $(r-J)$ as $(r-J)=(R-J)-(R-r)$. The solar color, with $g-r=0.45$ and $r-J=0.97$, is shown by the yellow star. The dashed curve indicates the reddening line, a line of constant spectral slope through the $g r J$ spectral range, calculated using the pysynphot software package (Lim et al. 2015). A broad trend of redness relative to the Sun exists, but detail within the population cannot be discerned.

\subsection{Target TNO Selection}

All Col-OSSOS targets are drawn from the sample of over 800 TNOs and Centaurs ( $\left.m_{r}=21.8-25.2 \mathrm{mag} ; 3.6<H_{r}<14.5 \mathrm{mag}\right)$ found by the OSSOS (Bannister et al. 2016, 2018), a survey with well-characterized survey biases. OSSOS was a wide-field $r$-band survey with the $3.58 \mathrm{~m}$ Canada-France-Hawaii Telescope's (CFHT) MegaPrime square-degree field-of-view (FOV) imager (MegaCam; Boulade et al. 2003). Operating from 2013 to 2017, OSSOS searched eight $20 \mathrm{deg}^{2}$ regions (blocks) of sky (Bannister et al. 2018), at locations where resonant TNOs come to perihelion and become optimally detectable. Strong emphasis was placed on thorough recovery and tracking of discoveries in a dense observing cadence across $2 \mathrm{yr}$, freeing the discovered TNOs from the challenges of ephemeris bias (Jones et al. 2006). A total of $97 \%$ of the OSSOS TNO sample has fractional semimajor axis uncertainty of $\sigma_{a}<0.1 \%$ (Bannister et al. 2016, 2018). The biases that surveying imposed on the TNO sample are well quantified for OSSOS discoveries (see Bannister et al. 2016; Lawler et al. 2018a). The high-quality orbit dynamics and population statistics of OSSOS form a framework on which to overlay the color information provided by Col-OSSOS. The Col-OSSOS sample consists of the 96 TNOs brighter than or equal to $m_{r}=23.6$ in the $13 \mathrm{AE}, 13 \mathrm{AO}, 13 \mathrm{BL}, 14 \mathrm{BH}$, and 15BS OSSOS blocks. ColOSSOS photometry for the 35 TNOs from 13BL and 14BH blocks is reported in this paper (see Section 5).

\subsection{Filter Selection and Color Precision}

The compositional classes described in Section 2.4 are identified by clusters in the optical and NIR reflectance colors exhibited by TNOs. The predominantly linear spectra of small, spectrally featureless TNOs in optical wavelengths are fully characterized by their $g-r$ color (see Doressoundiram et al. 2007). The neutral/red bifurcation of the dynamically excited TNOs exists only in the optical and is most prominent in $(g-r)$ (Doressoundiram et al. 2008; Fraser \& Brown 2012). With observations at wavelengths longer than $I$, additional surface classes become apparent in other small-body populations (DeMeo et al. 2009; Emery et al. 2011). This is shown indirectly for TNOs by Doressoundiram et al. (2008), who present $B V R I$ photometry of $\sim 100$ sources, with only two surface types apparent. The transition from the optical to NIR spectral gradients occurs at $\sim 1 \mu \mathrm{m}$; thus, only filters at longer wavelengths can provide the necessary slope information. It should be noted that Dalle Ore et al. (2013) present no practical difference between the $J, H$, and $K$ bands in terms of identifying their proposed compositional classifications.

In order to distinguish between the compositional classes proposed by Dalle Ore et al. (2013) and Fraser \& Brown (2012), we selected two optical broadband filters, $g$ and $r$, and an NIR filter, $J$, for our observations. These were the fewest filters that could define the optical and NIR slope of each TNO in the wavelength region of interest; $(g-r)$ characterizes the optical slope, and $(r-J)$ characterizes the NIR slope. Figure 1 shows the color precision of all available optical $+J$-band color measurements as of the start of our survey (Hainaut et al. 2012; Peixinho et al. 2015), with appropriate conversions to $\mathrm{grJ}$ (Jester et al. 2005; Jordi et al. 2006). These measurements are mean values, which do not necessarily account for the rotational variability of each TNO. These data demonstrate the importance of precision and temporal near-simultaneity in color measurements: their precision is insufficient to distinguish the color classes discussed in Section 2.4. Those classes have optical and NIR colors that differ by as little as 0.04-0.06 mag from class to class. The photometric uncertainty in previously published color studies is in the range of $0.04-0.2 \mathrm{mag}$, with no measurements in the size/ $\mathrm{H}$ range that Col-OSSOS aimed to sample (Hainaut et al. 2012; Peixinho et al. 2015). To achieve $0.06 \mathrm{mag}$ or better photometric precision for the Col-OSSOS sample required the collecting area of an $8-10 \mathrm{~m}$ class telescope and the nonstandard observing and analysis techniques that we describe in Sections 3.3 and 4 and Appendices A and B.

As we observe in a filter system that is close to widely used bandpasses but has subtle distinctions worth accounting for at our required level of photometric precision, throughout this 
paper we use the following nomenclature for our filters and corresponding colors:

optical bandpasses, in contexts where the specific filter system does not need to be distinguished;

Maunakea Observatory (MKO) filter set $J$ band (Simons \& Tokunaga 2002), $\lambda=12500 \AA, 11500-13300 \AA$ coverage.

Where necessary for specific observations and color conversions, we specify the exact bandpass in $g, r$, or $z$ with appropriate subscripts, as shown here for $r$ :

- Sloan Digital Sky Survey (SDSS) Photometric System (Fukugita et al. 1996; Padmanabhan et al. 2008).

- Gemini Multi-Object Spectrograph (GMOS) North filters. ${ }^{18}$ Acquired with either the E2V or the Hamamatsu detectors (specified as needed; see Section 3.3).

- Pan-STARRS1 photometric system (Tonry et al. 2012).

\subsection{Observing Strategy}

Our primary observing facility is the $8.1 \mathrm{~m}$ Frederick C. Gillett Gemini North Telescope located on Maunakea, Hawai'i. In the optical, each target was observed with the imaging mode $(5.5 \times 5.5$ FOV $)$ of the Gemini Multi-Object Spectrograph (GMOS; Hook et al. 2004) using the r_G0303 $(\lambda=6300 \AA, \quad \delta \lambda=1360 \AA)$ and $g_{-} \operatorname{Go301}(\lambda=4750 \AA$, $\delta \lambda=1540 \AA$ ) filters, similar to the SDSS $r$ and $g$ bands. The GMOS observations were obtained in $1 \times 1$ binning mode. This had 0 " 0747 pixels with the $2 \mathrm{v}$ deep depletion charge-coupled devices (CCDs) available in GMOS during the 2014-2016 observing semesters, and 0."0807 pixels after the 2017 installation of Hamamatsu red-sensitive CCDs. GMOS observations were dithered by $3^{\prime \prime}-5^{\prime \prime}$ from exposure to exposure. For the NIR measurements, we observed with the Near-Infrared Imager (NIRI; Hodapp et al. 2003) in MKO J. NIRI observations were acquired using the $f / 6$ camera $(0$ " 116 per pixel resolution) with a $119 . ! 9 \times 119$ !" 9 FOV. NIRI observations utilized a grid dither pattern with $8^{\prime \prime}$ spacing between exposures to ensure accurate background measurement and removal. For both NIRI and GMOS, these instrument configurations allow the best possible characterization of the point-spread function (PSF), and hence the best knowledge of the photometric curve of growth.

For our color precision requirement to detect and distinguish the Dalle Ore et al. (2013) classes, we aim for a 0.06 mag color precision in $(g-r)$ and $(r-J)$ for all targets. To achieve our desired color precision (Section 3.2), we require a signal-tonoise ratio $(\mathrm{S} / \mathrm{N})$ of $\geqslant 25$ in $g$ and $r$, and $\mathrm{S} / \mathrm{N} \geqslant 20$ in $J$. For each Col-OSSOS TNO, the total effective exposure time required in each filter was estimated using the mean OSSOS $r$ band discovery magnitude and assuming very red optical and only modestly red NIR colors relative to those typically exhibited by small TNOs (Figure 1): $g-r=1.1$ and $r-J=1.2$. Ideally, most TNOs will have bluer optical colors and redder NIR colors, and their observations will thus meet our $\mathrm{S} / \mathrm{N}$ requirements regardless of their true color. At the time of the Gemini observations no light curves were yet measured for these TNOs. If the 15-20 OSSOS CFHT observations of the TNO over $1-2$ yr showed significant \pm 0.3 mag photometric variability, additional frames in all filters were added to the

\footnotetext{
18 See http://www.gemini.edu/node/10420.
}

Gemini observing sequence, in case observing happened during minimum TNO brightness.

We used Gemini's fast instrument switching abilities ( $\lesssim 4$ minutes) to provide near-simultaneous colors: all targets were visited in an unbroken imaging sequence of rgJgr. Anchoring the sequence with $r$ exposures lets us account for light-curve variations during color estimates (discussed further in Section 4). Observations were executed to gather the desired cumulative $\mathrm{S} / \mathrm{N}$ by taking half the required optical frames before and then after the required $J$-band observations. Individual GMOS exposures were set to $300 \mathrm{~s}$ in duration. Individual NIRI exposures were limited to $120 \mathrm{~s}$, both to minimize trailing losses and to mitigate the high sky background.

The telescope tracked at the sidereal rate, permitting use of calibration stars within the images (see Appendices A and B). Source trailing in each observation was minimal, as ColOSSOS targets typically have on-sky motions of several arcseconds per hour or less. The timing of the observations was chosen to avoid the TNO passing over or close to bright or contaminating background stars and galaxies. A combination of SDSS observations (York et al. 2000), stacked OSSOS images (Bannister et al. 2018), and stacked Pan-STARRS1 (Chambers et al. 2016; Flewelling et al. 2016) images was used to identify times when the TNOs were moving through areas of sky devoid of background sources brighter than $\sim 24$ th magnitude. The majority of the GMOS observations were made at an airmass $<2$, and most of the NIRI photometry was obtained at an airmass $<1.4$. With Gemini's queue scheduling and priority visitor mode, all observations were made in photometric conditions during dark time, in Gemini's 50th percentile sky background (SB 50) criteria. The images were executed in Gemini's 70th percentile image quality (IQ 70) or better, achieving image quality typically 0.7 or better in the optical and 0.15 or better in the NIR for the majority of observations. Before and after each $r g J g r$ sequence, NIRI photometric calibrator frames were acquired with bright standard stars at different elevations, chosen to encompass the range of elevations spanned during the $J$ observations of each TNO. Exposure times for the calibration stars were chosen so as not to saturate the NIRI detector, and exposures were taken in a nine-point dither pattern sequence. Associated NIRI lamp flats, NIRI dark frames, and GMOS bias observations were also obtained for each night of observing.

\subsection{1. z-band Imaging}

A subsample of the Col-OSSOS targets were also imaged with Gemini in the $z_{-} G 0304$ filter (8500-10000 $\AA$ coverage). The inclusion of $z$ observations was ad hoc and with no predefined minimum $\mathrm{S} / \mathrm{N}$ requirement. During exceptional sky conditions (IQ 20, c.f. $\sim 0$ ". 4 seeing), we reduced the number of $g$ and $r$ frames, maintaining $\mathrm{S} / \mathrm{N}=25$, and added GMOS $z_{\mathrm{G}}$-band observations. They bracketed the start and end of the optical sequences, with the same $300 \mathrm{~s}$ exposure time. We describe the $z_{\mathrm{G}}$ data processing in Appendix $\mathrm{A}$ and their implications in Pike et al. (2017). Additional simultaneous $z$ images were acquired with Suprime-Cam (Miyazaki et al. 2002) on the Subaru Telescope in 2014 August. These observations are reported in detail in Pike et al. (2017). 


\section{TNO Color Technique}

Detailed overviews of the optical and NIR data reduction, photometry, and calibration we performed on Col-OSSOS observations are described in Appendices A and B. Photometry was performed on each individual GMOS frame. For the NIRI observations, the sequences of images were divided and combined into two stacked images; photometry was performed on each. Our measurements were made with TRIPPy (Trailed Image Photometry in Python), a dedicated software for photometry of linearly trailed sources (Fraser et al. 2016). TRIPPy makes use of a pill-shaped aperture, an aperture elongated based on a solar system object's predicted rate of motion. For trailed solar system sources, this process correctly accounts for the flux that would be lost in making use of circular apertures, while maintaining the photometric precision found with use of small-area apertures. For PSFs derived from sidereal tracked stars, aperture corrections can be determined to better than $0.01 \mathrm{mag}$ for the pill aperture (Fraser et al. 2016). Our optical and NIR measurements were calibrated to the SDSS (York et al. 2000; Padmanabhan et al. 2008) filter system and the MKO filter set $J$ band (Simons \& Tokunaga 2002), respectively. The estimation of the color transform between the SDSS and Gemini filter sets is described in Appendix A.3.

The final step to estimating colors is to account for brightness variations of the TNO over the duration of the science sequence. Any significant amplitude changes due to rotational variability of the TNO can cause difficulty combining measured broadband colors if not accounted for. Our full GMOS-NIRI-GMOS sequences span between 1 and $6 \mathrm{hr}$. Small TNOs $(H>5)$ typically have $6-15 \mathrm{hr}$ rotation periods, with peak-to-peak variations of $\sim 0.3 \mathrm{mag}$ (Trilling \& Bernstein 2006; Duffard et al. 2009; Benecchi \& Sheppard 2013), though significantly larger variations have been observed (Fraser et al. 2015; Thirouin \& Sheppard 2018), including in the OSSOS sample (Alexandersen et al. 2018). ${ }^{19}$

We took advantage of each sequence's bracketing $g$ and $r$ imagery to correct for light curves by fitting a linear model to all optical data. An example of a fitted light curve for a target that exhibited a variation in brightness over the Col-OSSOS observing sequence is presented in Figure 2. This model makes two assumptions: that the light-curve variations observed across a given sequence are linear in nature, and that the object exhibits no significant color variations across the sequence. Thus, the model has the following free parameters: slope (change in brightness with time), a reference $r$ magnitude, a color $g-r$, and sometimes a color $r-z$, where $z$ images were available. This model was fit in a least-squares sense to the available data. To evaluate uncertainties on each optical color, we adopt a Monte-Carlo approach. Specifically, each individual photometric measurement was scattered by a Gaussian distribution with width equal to the photometric uncertainty on that point, and the scattered data set was fit. This process was repeated 200 times, and the quadrature sum of the standard deviation on the randomized color terms and the uncertainty on the mean of the individual photometric measurements were adopted as the uncertainty on those points. The fitted linear light curve was then used to estimate the $r$ brightness at the midpoint of each $J$-band measurement so as to

\footnotetext{
19 Alexandersen et al. (2018) examine the photometric variability for four targets in our first-release sample: 2013 UL15, 2013 UP15, 2013 UM15, and 2013 UN15.
}

determine the mean $r-J$ color. Uncertainties in this color include the photometric uncertainties on $J$ and the uncertainties due to the light-curve model parameters (both slope and reference $r$ value).

We note that the linear fitting process for intrinsic variability will only correctly remove any first derivatives in the light curve. It will not account for the full range of possible photometric variation. We are not able to correct for, nor are we able to meaningfully estimate the additional uncertainty in, our color estimates caused by these unknown nonlinear light-curve variations. There are undoubtedly shape and albedo effects that impact our color estimates that are not accounted for in the linear fit. However, with the small number of photometric measurements we have for each target, we have no resolution to further constrain these effects. We discuss the impact of nonlinear light-curve variations on our results in Section 5.2.2.

\section{Col-OSSOS First Release: The OSSOS 13BL and 14BH Sample}

The observations we present here comprise a complete fluxlimited sample of TNOs: all the $m_{r}<23.6 \mathrm{mag}$ discoveries from the 13BL and 14BH OSSOS survey blocks (Bannister et al. 2016, 2018). In this section, we also present the orbital properties of our TNO release sample and summarize specific observing circumstances and data analysis unique to these targets compared to the overall Col-OSSOS survey strategy.

\subsection{Observational and Orbital Properties of the TNO Sample from 13BL and 14BH Blocks}

The 35 TNOs in this first Col-OSSOS release were found in 2013 and 2014 in two OSSOS survey regions of sky near the ecliptic, in a latitude range from the invariant plane up to $5^{\circ}$ off-plane. They comprise the $18 m_{r}<23.6 \mathrm{mag}$ discoveries from the 13BL block of OSSOS (o3l-designated targets) and the $17 m_{r}<23.6$ mag TNOs from the 14BH block of OSSOS (o4h-designated targets). 13BL block is a $20 \mathrm{deg}^{2}$ region overlaying the invariant plane, centered at R.A. $0^{\mathrm{h}} 54^{\mathrm{m}}$, decl. $+3^{\circ} 50^{\prime}$. 14BH block is a $21 \mathrm{deg}^{2}$ region $2^{\circ}-5^{\circ}$ off the invariant plane, centered at R.A. $1^{\mathrm{h}} 35^{\mathrm{m}}$, decl. $+13^{\circ} 28^{\prime}$. The detection efficiency of OSSOS for moving objects in each survey region is thoroughly characterized (Bannister et al. 2018).

Table 1 lists the orbital information for the 35 TNOs. The orbital distribution is shown in Figure 3. We tally the dynamical classes in Table 2. The TNOs in this first ColOSSOS release predominantly sample the classical Kuiper Belt and the resonant populations. The barycentric orbital properties of the 35 TNOs are derived from 2 to $5 \mathrm{yr}$ of densely sampled observations (Bannister et al. 2018). A full search for binarity of our sample is beyond the scope of this work, but Fraser et al. (2017) identified three cold classicals in our sample as binaries: 2016 BP81, 2014 UD225, and 2013 SQ99. One hot classical Kuiper Belt object, 2013 UQ15, has a semimajor axis, inclination, and eccentricity consistent with being in the dynamical cloud of the Haumea collisional family (Brown et al. 2007; Ragozzine \& Brown 2007). Five of the 14 resonant TNOs are in 3:2 mean motion resonance with Neptune, with the rest in 5:3, 4:3, 7:4, 5:2, 11:6, and 9:5 resonances. One TNO, 2007 TC434, is securely in the most distant resonance with Neptune yet confirmed, 9:1 at $a \sim 130$ au (Volk et al. 2018). There are also three objects from the more transient Centaur and scattering populations. The majority of our TNO 


\section{UK255}

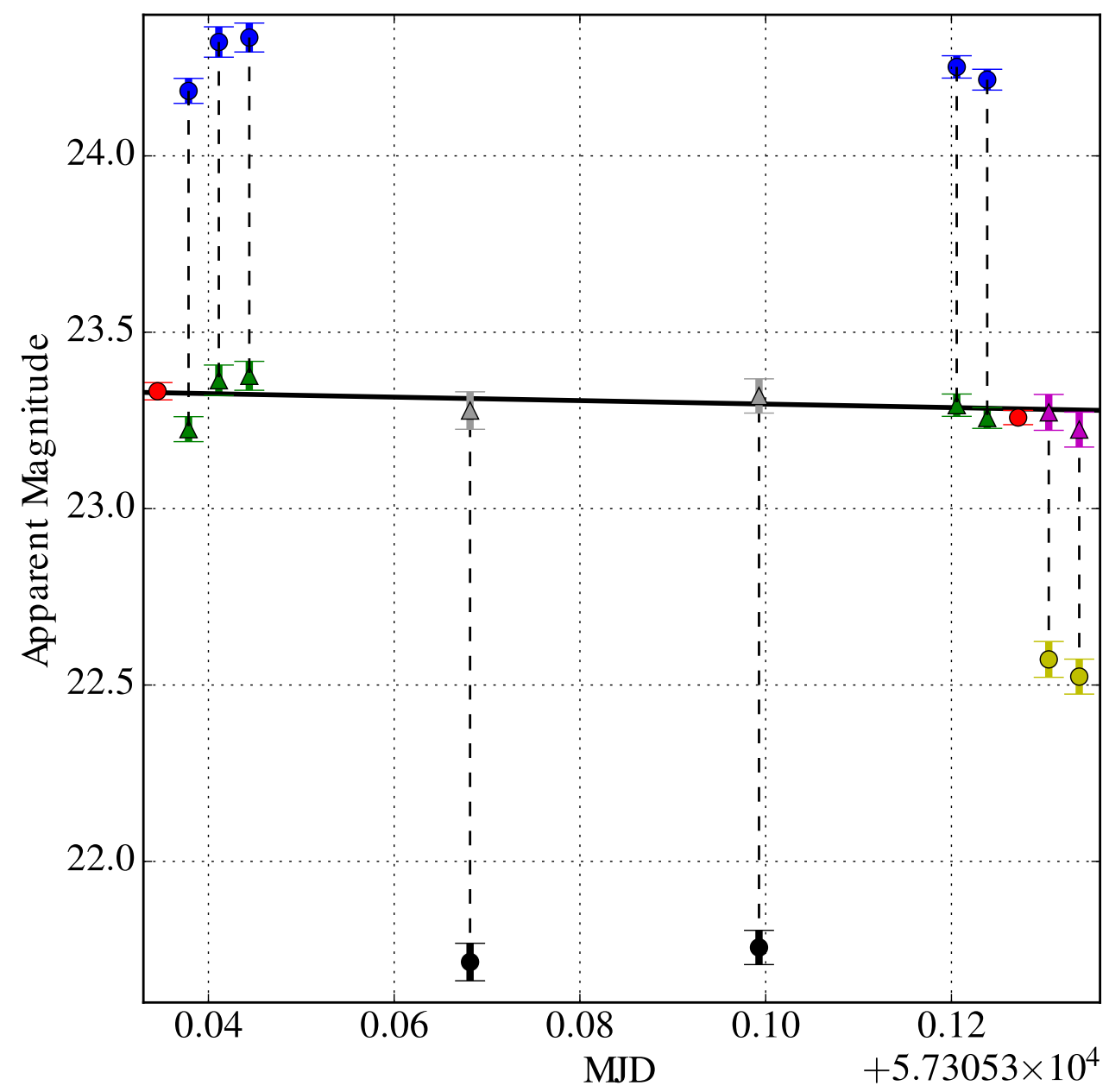

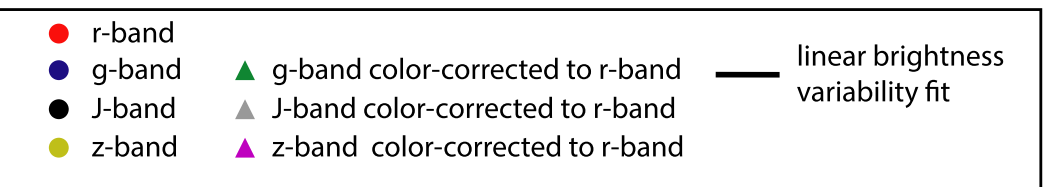

Figure 2. Example of observed photometry, fitted light curve, and colors for object 2014 UK225. Observed $r, g, z$, and $J$ measurements are shown by circles. Those points adjusted to $r$ band based on the fitted colors are plotted as triangles. The target exhibits an $\sim 0.06$ mag increase in brightness over the duration of the sequence. This change in brightness is comparable to the precision in measured colors of the source and is accounted for by our light-curve fitting technique.

sample consists of new discoveries by OSSOS. No targets in our release sample have previous near-simultaneous multifilter photometry to the precision that we discuss here. Of the few targets with observations earlier than when OSSOS began in 2013, none have published colors (Hainaut et al. 2012; J. J. Kavelaars 2019, private communication).

All of our 35 targets have absolute magnitudes $H_{r}>5$ and are thus much smaller than the dwarf planet size transition of $H \simeq 4$ (Brown 2008; Tancredi \& Favre 2008). Most have $5<H_{r}<8$, brighter than the break in the measured TNO luminosity function at $H_{r} \sim 8$ (Bernstein et al. 2004; Fuentes \& Holman 2008; Fraser \& Kavelaars 2009; Adams et al. 2014; Fraser et al. 2014). Only two have $H_{r}>10$.

\subsection{Observations and Data Analysis}

The Gemini observations were acquired as described in Section 3.3, during 2014-2015 (under programs GN-2014BLP-1, GN-2015A-LP-1, and GN-2015B-LP-1), with some minor exceptions described below. We also note here any details where the specific analysis differed from the general analysis for the whole survey given in Section 4 and Appendices A and B. Table 3 gives a detailed summary of the GMOS-N and NIRI observations for each target TNO. For all observations, GMOS-N was equipped with the e2v deep depletion detectors. Table 3 lists the calculated SDSS magnitude of the observed TNO and associated Gemini filter zero-point for each GMOS and NIRI exposure, as detailed in Appendix A.3. We note that our reported uncertainty in the optical magnitudes combines the uncertainties in the SDSS transformation, calculated zero-point, and flux measurement. The full photometry sequences for all target TNOs in this paper are presented in the supplemental material, and a representative sample is plotted in Appendix C.

Exceptional sky conditions occurred during the 2014 August priority visitor run, where many observations were acquired in 0 ". $4-0$ "! 5 seeing. The 2015 October priority visitor run had IQ 
Table 1

Orbital Parameters and Optical and NIR Colors of the o31 and o4h Col-OSSOS TNO Sample

\begin{tabular}{|c|c|c|c|c|c|c|c|c|c|c|c|c|}
\hline $\begin{array}{l}\text { MPC } \\
\text { ID }\end{array}$ & $\begin{array}{l}\text { OSSOS } \\
\text { ID }\end{array}$ & $\begin{array}{c}a \\
(\mathrm{au})\end{array}$ & $e$ & $\begin{array}{l}\text { Inc } \\
\text { (Deg) }\end{array}$ & $\begin{array}{c}\Delta \\
(\mathrm{au})\end{array}$ & $\begin{array}{l}r_{\text {helio }} \\
(\mathrm{au})\end{array}$ & $\begin{array}{c}\text { Mean } m_{r} \\
\text { (SDSS) }\end{array}$ & $H_{r}$ & $\begin{array}{l}\text { Orbit } \\
\text { Class }\end{array}$ & $\begin{array}{l}g-r \\
\text { (SDSS) }\end{array}$ & $\begin{array}{c}r-J \\
\text { (SDSS-MKO) }\end{array}$ & $\begin{array}{c}r-z \\
\text { (SDSS) }\end{array}$ \\
\hline $2013 \mathrm{UR} 5^{\mathrm{a}}$ & 03101 & 55.82 & 0.719 & 22.25 & 15.69 & 16.31 & $23.40 \pm 0.17$ & 11.36 & sca & $0.67 \pm 0.02$ & $1.49 \pm 0.08$ & $\cdots \pm \cdots$ \\
\hline $2001 \mathrm{QF} 331^{\mathrm{a}, \mathrm{b}}$ & o3106PD & 42.25 & 0.252 & 2.67 & 31.88 & 32.60 & $22.92 \pm 0.03$ & 7.84 & $5: 3$ & $0.88 \pm 0.03$ & $1.67 \pm 0.05$ & $\cdots \pm \cdots$ \\
\hline 2013 US15 $^{\mathrm{a}}$ & o3109 & 36.38 & 0.070 & 2.02 & 33.66 & 34.40 & $23.17 \pm 0.02$ & 7.85 & $4: 3$ & $1.03 \pm 0.02$ & $1.49 \pm 0.05$ & $\cdots \pm \cdots$ \\
\hline 2003 SR317 & o3113PD & 39.43 & 0.166 & 8.35 & 36.27 & 37.23 & $23.42 \pm 0.08$ & 7.77 & $3: 2$ & $0.64 \pm 0.01$ & $1.33 \pm 0.05$ & $\cdots \pm \cdots$ \\
\hline $2013 \mathrm{SZ}^{\mathrm{a}}$ & 03115 & 38.28 & 0.017 & 19.84 & 37.96 & 38.75 & $23.80 \pm 0.06$ & 7.96 & cla & $0.68 \pm 0.02$ & $1.35 \pm 0.07$ & $\cdots \pm \cdots$ \\
\hline 2010 RE188 & o3118 & 46.01 & 0.147 & 6.75 & 38.90 & 39.64 & $22.34 \pm 0.02$ & 6.40 & cla & $0.58 \pm 0.02$ & $1.43 \pm 0.06$ & $\cdots \pm \cdots$ \\
\hline 2013 SP99 $^{\mathrm{c}}$ & 03132 & 43.78 & 0.060 & 0.79 & 41.09 & 42.01 & $23.53 \pm 0.05$ & 7.35 & cla & $1.00 \pm 0.02$ & $1.61 \pm 0.05$ & $\cdots \pm \cdots$ \\
\hline 2016 BP81 $1^{\mathrm{a}, \mathrm{c}, \mathrm{d}}$ & 03139 & 43.68 & 0.076 & 4.18 & 41.81 & 42.54 & $22.83 \pm 0.11$ & 6.58 & $7: 4 I$ & $0.59 \pm 0.03$ & $1.60 \pm 0.07$ & $\cdots \pm \cdots$ \\
\hline 2013 UL15 $^{\mathrm{a}, \mathrm{b}, \mathrm{c}}$ & o3143 & 45.79 & 0.097 & 2.02 & 42.38 & 43.10 & $23.12 \pm 0.10$ & 6.82 & cla & $0.90 \pm 0.04$ & $1.51 \pm 0.06$ & $\cdots \pm \cdots$ \\
\hline $2013 \mathrm{UP} 15^{\mathrm{a}, \mathrm{c}}$ & o3146 & 46.61 & 0.079 & 2.47 & 42.70 & 43.38 & $23.92 \pm 0.10$ & 7.58 & cla & $0.90 \pm 0.02$ & $1.78 \pm 0.07$ & $\cdots \pm \cdots$ \\
\hline $2013 \mathrm{UO}^{\mathrm{c}}{ }^{\mathrm{c}}$ & o3150 & 43.33 & 0.049 & 3.73 & 43.08 & 43.99 & $23.28 \pm 0.00$ & 6.89 & cla & $0.96 \pm 0.02$ & $1.75 \pm 0.04$ & $\cdots \pm \cdots$ \\
\hline $2013 \mathrm{UM} 15^{\mathrm{b}} \mathrm{c}$ & 03157 & 45.04 & 0.075 & 1.84 & 43.77 & 44.46 & $23.39 \pm 0.00$ & 6.95 & $11: 6$ & $1.08 \pm 0.01$ & $1.56 \pm 0.06$ & $\cdots \pm \cdots$ \\
\hline $2006 \mathrm{QF}^{2} 1^{\mathrm{c}}$ & o3160 & 44.82 & 0.076 & 2.66 & 44.20 & 44.54 & $23.56 \pm 0.02$ & 7.08 & cla & $0.89 \pm 0.03$ & $1.65 \pm 0.05$ & $\cdots \pm \cdots$ \\
\hline $2013 \mathrm{UN}^{2} 5^{\mathrm{a}, \mathrm{c}, \mathrm{e}}$ & o3163 & 45.13 & 0.054 & 3.36 & 44.39 & 45.14 & $24.13 \pm 0.09$ & 7.62 & cla & $1.04 \pm 0.03$ & $1.57 \pm 0.08$ & $0.37 \pm 0.09$ \\
\hline $2013 \mathrm{UN}^{\mathrm{a}} 5^{\mathrm{a}, \mathrm{c}, \mathrm{f}, \mathrm{g}}$ & o3163 & 45.13 & 0.054 & 3.36 & 44.20 & 45.19 & $23.62 \pm 0.00$ & 7.11 & cla & $1.08 \pm 0.03$ & $1.82 \pm 0.04$ & $0.73 \pm 0.06$ \\
\hline 2013 UX18 & o3169 & 43.60 & 0.057 & 2.89 & 44.78 & 45.73 & $23.93 \pm 0.00$ & 7.37 & cla & $0.93 \pm 0.01$ & $1.64 \pm 0.08$ & $\cdots \pm \cdots$ \\
\hline $2013 \mathrm{SQ}^{\mathrm{a}, \mathrm{c}, \mathrm{d}}$ & 03176 & 44.15 & 0.093 & 3.47 & 46.60 & 47.34 & $23.17 \pm 0.04$ & 6.45 & cla & $0.98 \pm 0.03$ & $1.51 \pm 0.06$ & $0.56 \pm 0.03$ \\
\hline $2013 \mathrm{UQ}^{\mathrm{a} 5^{\mathrm{a}, \mathrm{b}} \mathrm{h}}$ & o3177 & 42.77 & 0.113 & 27.34 & 46.84 & 47.54 & $23.02 \pm 0.22$ & 6.28 & cla & $0.53 \pm 0.04$ & $0.92 \pm 0.11$ & $\cdots \pm \cdots$ \\
\hline $2013 \mathrm{SA} 100^{\mathrm{a}, \mathrm{e}}$ & 03179 & 46.30 & 0.166 & 8.48 & 49.69 & 50.43 & $23.02 \pm 0.03$ & 6.03 & cla & $0.63 \pm 0.02$ & $1.66 \pm 0.08$ & $0.44 \pm 0.02$ \\
\hline $2013 \mathrm{SA}^{200}{ }^{\mathrm{a}, \mathrm{g}}$ & 03179 & 46.30 & 0.166 & 8.48 & 49.30 & 50.29 & $22.78 \pm 0.03$ & 5.81 & cla & $0.67 \pm 0.02$ & $1.48 \pm 0.04$ & $0.42 \pm 0.02$ \\
\hline $2014 \mathrm{UJ}^{2} 25^{\mathrm{a}}$ & o4h01 & 23.20 & 0.378 & 21.32 & 17.26 & 17.83 & $23.05 \pm 0.08$ & 10.61 & cen & $0.65 \pm 0.01$ & $1.08 \pm 0.09$ & $\cdots \pm \cdots$ \\
\hline 2014 UQ229 & o4h03 & 49.90 & 0.779 & 5.68 & 20.84 & 21.83 & $22.80 \pm 0.13$ & 9.51 & sca & $1.03 \pm 0.02$ & $1.94 \pm 0.05$ & $\cdots \pm \cdots$ \\
\hline 2014 UX229 & o4h05 & 39.63 & 0.335 & 15.97 & 25.76 & 26.41 & $22.50 \pm 0.07$ & 8.34 & $3: 2$ & $0.64 \pm 0.02$ & $1.53 \pm 0.06$ & $\cdots \pm \cdots$ \\
\hline 2010 TJ182 & o4h07 & 39.65 & 0.276 & 9.50 & 27.88 & 28.86 & $22.55 \pm 0.02$ & 8.02 & $3: 2$ & $0.60 \pm 0.02$ & $1.37 \pm 0.04$ & $\cdots \pm \cdots$ \\
\hline 2014 UV228 & o4h09 & 39.49 & 0.228 & 10.13 & 30.78 & 31.75 & $23.57 \pm 0.05$ & 8.62 & $3: 2$ & $0.65 \pm 0.02$ & $1.51 \pm 0.04$ & $\cdots \pm \cdots$ \\
\hline 2014 UO229 & o4h11 & 39.45 & 0.161 & 10.09 & 33.77 & 34.03 & $23.82 \pm 0.03$ & 8.52 & $3: 2$ & $0.72 \pm 0.02$ & $1.15 \pm 0.06$ & $\cdots \pm \cdots$ \\
\hline 2014 UD229 & o4h13 & 36.39 & 0.145 & 6.85 & 33.58 & 34.31 & $23.66 \pm 0.02$ & 8.35 & $4: 3$ & $0.71 \pm 0.02$ & $1.23 \pm 0.06$ & $\cdots \pm \cdots$ \\
\hline 2014 US229 & o4h14 & 55.26 & 0.398 & 3.90 & 32.32 & 33.31 & $23.47 \pm 0.01$ & 8.31 & $5: 2$ & $0.63 \pm 0.02$ & $1.50 \pm 0.05$ & $\cdots \pm \cdots$ \\
\hline 2014 UX228 & o4h18 & 36.35 & 0.167 & 20.66 & 37.01 & 37.99 & $23.20 \pm 0.03$ & 7.46 & $4: 3$ & $0.56 \pm 0.03$ & $1.44 \pm 0.05$ & $\cdots \pm \cdots$ \\
\hline 2014 UK $225^{\mathrm{a}}$ & o4h19 & 43.52 & 0.127 & 10.69 & 37.09 & 38.06 & $23.32 \pm 0.04$ & 7.57 & cla & $0.95 \pm 0.02$ & $1.60 \pm 0.04$ & $0.69 \pm 0.02$ \\
\hline $2014 \mathrm{UL}_{225^{\mathrm{a}}}$ & $\mathrm{o} 4 \mathrm{~h} 20$ & 46.34 & 0.199 & 7.95 & 37.36 & 37.96 & $23.33 \pm 0.11$ & 7.57 & cla & $0.55 \pm 0.03$ & $1.03 \pm 0.09$ & $\cdots \pm \cdots$ \\
\hline $2014 \mathrm{UH} 225^{\mathrm{a}}$ & o4h29 & 38.64 & 0.037 & 29.53 & 39.08 & 40.06 & $23.48 \pm 0.11$ & 7.50 & cla & $0.57 \pm 0.02$ & $1.69 \pm 0.04$ & $0.38 \pm 0.03$ \\
\hline $2014 \mathrm{UM}^{2} 25^{\mathrm{a}}$ & $\mathrm{o} 4 \mathrm{~h} 31$ & 44.48 & 0.098 & 18.30 & 39.50 & 40.16 & $23.53 \pm 0.06$ & 7.52 & $9: 5$ & $0.80 \pm 0.02$ & $1.60 \pm 0.04$ & $\cdots \pm \cdots$ \\
\hline 2007 TC434 & o4h39 & 129.92 & 0.695 & 26.47 & 39.74 & 40.60 & $23.47 \pm 0.05$ & 7.43 & $9: 1$ & $0.64 \pm 0.02$ & $1.59 \pm 0.05$ & $\cdots \pm \cdots$ \\
\hline 2014 UD225 $5^{\mathrm{a}, \mathrm{c}, \mathrm{d}}$ & $\mathrm{o} 4 \mathrm{~h} 45$ & 43.36 & 0.130 & 3.66 & 43.67 & 44.29 & $22.98 \pm 0.05$ & 6.55 & cla & $0.74 \pm 0.02$ & $1.42 \pm 0.06$ & $\cdots \pm \cdots$ \\
\hline 2001 RY143 & $\mathrm{o} 4 \mathrm{~h} 48$ & 42.08 & 0.155 & 6.91 & 46.34 & 47.32 & $23.66 \pm 0.08$ & 6.95 & cla & $0.92 \pm 0.03$ & $1.88 \pm 0.06$ & $\cdots \pm \cdots$ \\
\hline 2014 UE $225^{\mathrm{a}, \mathrm{c}}$ & o4h50 & 43.71 & 0.066 & 4.49 & 45.96 & 46.56 & $22.92 \pm 0.01$ & 6.27 & cla & $1.03 \pm 0.02$ & $1.87 \pm 0.05$ & $\cdots \pm \cdots$ \\
\hline
\end{tabular}

Notes. Orbit Class: dynamical classification of barycentric orbits from a $10 \mathrm{Myr}$ integration: cen $=$ Centaur, $\mathrm{sca}=$ scattering disk, cla $=$ classical belt, $N: M=$ mean motion resonance with Neptune. An "I" after the resonant identifier signifies an insecure resonance classification. See Bannister et al. (2018) for further details. Geometric parameters and derived $H_{r}$ are reported for the time of the Col-OSSOS observation (see Table 3). Mean $m_{r}$ is the mean of the measured Col-OSSOS $r$-band photometry. Values for individual frames are reported in Table 3. Table 1 is published in a machine-readable format. Those targets with two observation epochs have a separate entry for the color measurements derived at each epoch, ordered chronologically.

a Targets with previously published optical colors in Pike et al. (2017) reprocessed here using the latest version of TRIPPy (Fraser et al. 2016), the most recent data analysis pipeline, and improved SDSS color terms. Additional Subaru $r$ and $z$ photometry for some of the highlighted targets is reported in Pike et al. (2017). Only $(r-z)$ colors obtained from Gemini observing sequences if available are reported here.

b Telescope tracked nonsidereally at the TNO's rate of motion on-sky.

${ }^{\mathrm{c}}$ Targets with previously published optical spectral slopes in Fraser et al. (2017) reprocessed here using the latest version of TRIPPy (Fraser et al. 2016), the most recent data analysis pipeline, and improved SDSS color terms.

${ }^{\mathrm{d}}$ Confirmed as a binary in Fraser et al. (2017).

e 2014B observations.

${ }^{\mathrm{f}}$ Photometry includes CFHT measurements.

g 2015B observations.

${ }^{\mathrm{h}}$ Orbit consistent with the Haumea collisional family cluster.

(This table is available in machine-readable form.)

$20(0$ ". $3-0$ ". 6 seeing). When observing in IQ 20 conditions, we shortened the individual GMOS frames to be less than $300 \mathrm{~s}$ for 10 TNOs (2001 RY143, 2010 RE188, 2010 TJ182, 2013 UR15, 2014 UE225, 2014 UH225, 2014 UK225, 2014 UL225, 2014 UV228, and 2016 BP81) while preserving our desired $\mathrm{S} / \mathrm{N}$ goals.
Two TNOs in the sample (2013 SA100 and 2013 UN15) were observed twice. We report both color measurements in Tables 1 and 3. The dynamically excited TNO 2013 SA100 was observed in both the 2014B and 2015B semesters, with a full $g r z J$ sequence at each epoch. The cold classical 2013 UN15 was observed in both the 2014B and 2015B semesters. 

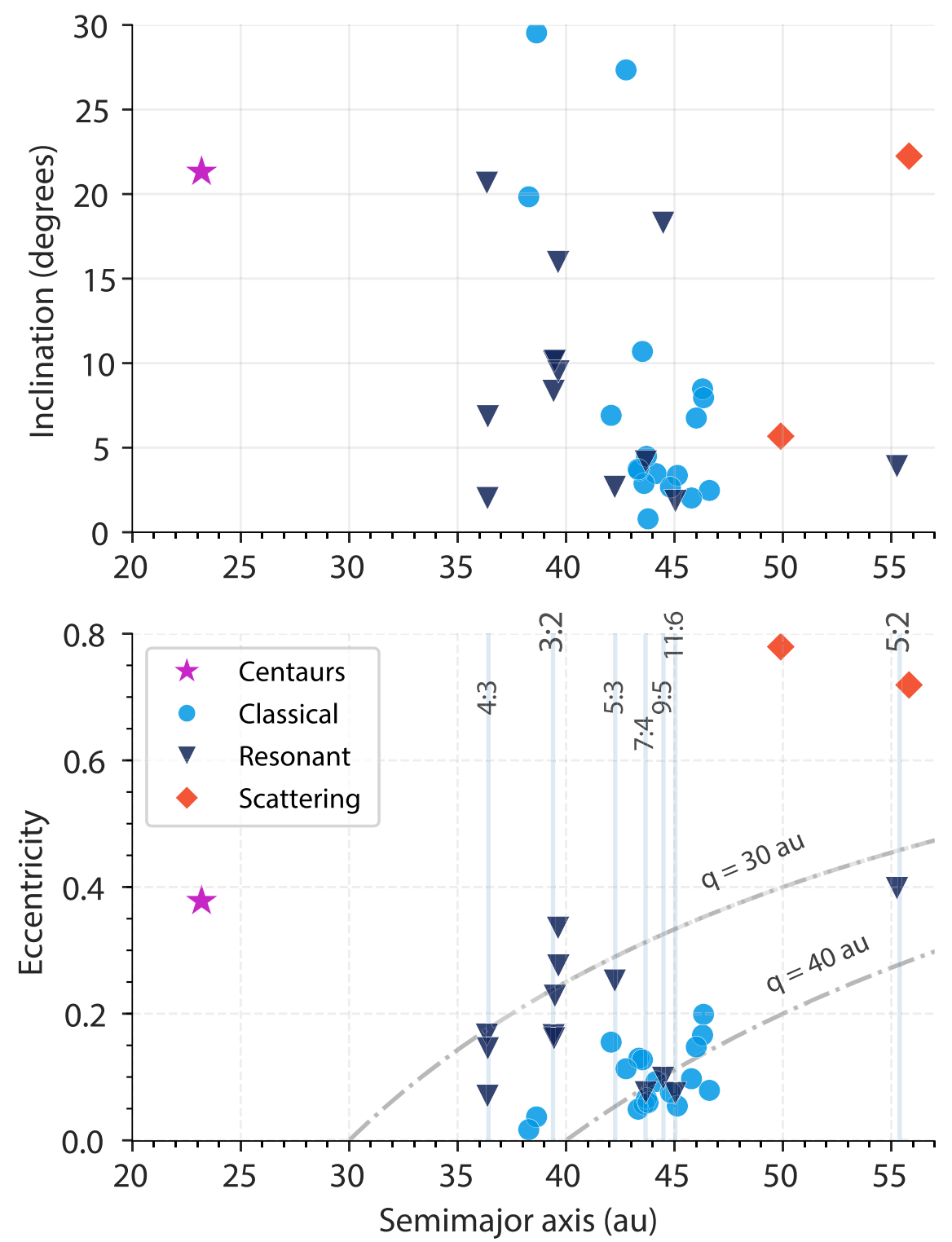

Figure 3. Barycentric orbital parameters, derived from Bannister et al. (2018), of TNOs with Col-OSSOS color measurements presented in this paper. One TNO at $a=130 \mathrm{au}$ is omitted for better resolution. The $1 \sigma$ uncertainties are smaller than the size of the plot symbol.

Table 2

Dynamical Classifications of the Col-OSSOS 13BL and 14BH Block Targets

\begin{tabular}{lcc}
\hline \hline Orbital Class & $\#$ & Comment \\
\hline Centaurs & 1 & \\
Cold classicals & 10 & $i<5^{\circ}$ inclination, all in main belt \\
Hot classicals & 8 & 1 consistent with the Haumea family \\
Resonant & 14 & $3: 2,5: 3,4: 3,7: 4,{ }^{\text {a }} 5: 2,11: 6,9: 5,9: 1$ \\
Scattering & 2 & \\
\hline
\end{tabular}

Note.

a $7: 4$ resonance identification of 2016 BP81 is insecure. See Bannister et al. (2018) for further details.

As it appeared very red in the 2014B observations $(r-J \sim$ 1.5; Table 1), additional time was spent on the $2015 J$ observations, with no Gemini $g$ observations. The $g$ observations were instead acquired simultaneously by MegaCam on CFHT with an $r g r$ sequence of $10300 \mathrm{~s}$ g.MP9402 filter images bracketed by two $300 \mathrm{~s}$ r.MP9602 filter images before and after. We also report the CFHT photometry and calculated zero-points in Table 3.

For 4 of the 35 objects (2001 QF331, 2013 UL15, 2013 UM15, and 2013 UQ15), the telescope instead tracked at the on-sky rate of motion for the target TNO rather than standard sidereal tracking. Given their slow rate of motion of a few arcseconds per hour, the PSFs of both the stars and TNO were still quite round in the observations. Thus, the photometry was measured with the same procedure as the sidereally tracked targets, but the uncertainty in the aperture correction was doubled to 0.02 mag to reflect the small errors induced by the nonsidereal tracking. Additionally, Fraser et al. (2017) identified three of the cold classicals in our sample as binaries: 2016 BP81, 2013 SQ99, and 2014 UD225. In our GMOS observations, the objects were elongated, but the components were not fully separated. Photometric apertures with radii of $2.5 \times$ FWHM and appropriate aperture corrections were used to ensure that the flux of both sources was included in the aperture, for a combined photometric measurement (Table 3). 


\begin{tabular}{|c|c|c|c|c|c|c|c|c|c|}
\hline $\begin{array}{l}\text { MPC } \\
\text { ID }\end{array}$ & $\begin{array}{l}\text { OSSOS } \\
\text { ID }\end{array}$ & $\begin{array}{l}\text { Header } \\
\text { ID }\end{array}$ & $\begin{array}{l}\text { Reduced } \\
\text { Filename }\end{array}$ & Filter & MJD & Gemini mag & Zero-point & SDSS mag & $\begin{array}{c}\text { Exposure }^{\mathrm{a}} \\
\text { (s) }\end{array}$ \\
\hline 2013 UR15 & o3101 & O13BL3RQ & N20140825S0315.fits & r_G0303 & $56,894.43641$ & $23.234 \pm 0.022$ & $28.251 \pm 0.005$ & $23.275 \pm 0.022$ & 300 \\
\hline 2013 UR15 & 03101 & O13BL3RQ & N20140825S0316.fits & g_G0301 & $56,894.44091$ & $23.937 \pm 0.033$ & $28.136 \pm 0.005$ & $24.03 \pm 0.033$ & 300 \\
\hline 2013 UR15 & o3101 & O13BL3RQ & O13BL3RQ-0.fits & $\mathrm{J}$ & $56,894.46418$ & $21.953 \pm 0.117$ & $23.905 \pm 0.02$ & $\cdots \pm \cdots$ & 1200 \\
\hline 2013 UR15 & o3101 & O13BL3RQ & O13BL3RQ-1.fits & $\mathrm{J}$ & $56,894.47983$ & $21.912 \pm 0.094$ & $23.907 \pm 0.02$ & $\cdots \pm \cdots$ & 1320 \\
\hline 2013 UR15 & 03101 & O13BL3RQ & N20140825S0341.fits & g_G0301 & $56,894.49351$ & $24.12 \pm 0.035$ & $28.175 \pm 0.005$ & $24.213 \pm 0.036$ & 225 \\
\hline 2013 UR15 & o3101 & O13BL3RQ & N20140825S0342.fits & g_G0301 & $56,894.49706$ & $23.977 \pm 0.032$ & $28.18 \pm 0.006$ & $24.07 \pm 0.033$ & 225 \\
\hline 2013 UR15 & o3101 & O13BL3RQ & N20140825S0343.fits & r_G0303 & $56,894.50069$ & $23.584 \pm 0.029$ & $28.271 \pm 0.007$ & $23.625 \pm 0.029$ & 225 \\
\hline 2001 QF331 & o3106PD & O13BL3SH & N20140823S0289.fits & r_G0303 & $56,892.41022$ & $22.839 \pm 0.034$ & $28.22 \pm 0.005$ & $22.892 \pm 0.034$ & 300 \\
\hline 2001 QF331 & o3106PD & O13BL3SH & N20140823S0290.fits & g_G0301 & $56,892.41473$ & $23.702 \pm 0.039$ & $28.127 \pm 0.004$ & $23.825 \pm 0.039$ & 300 \\
\hline 2001 QF331 & o3106PD & O13BL3SH & N20140823S0291.fits & g_G0301 & $56,892.41916$ & $23.621 \pm 0.038$ & $28.133 \pm 0.005$ & $23.745 \pm 0.038$ & 300 \\
\hline 2001 QF331 & o3106PD & O13BL3SH & O13BL3SH_0.fits & $\mathrm{J}$ & $56,892.43259$ & $21.32 \pm 0.063$ & $23.852 \pm 0.02$ & $\cdots \pm \cdots$ & 840 \\
\hline 2001 QF331 & o3106PD & O13BL3SH & O13BL3SH-1.fits & $\mathrm{J}$ & $56,892.44375$ & $21.197 \pm 0.055$ & $23.851 \pm 0.02$ & $\cdots \pm \cdots$ & 960 \\
\hline 2001 QF331 & o3106PD & O13BL3SH & N20140823S0308.fits & g_G0301 & $56,892.45469$ & $23.716 \pm 0.037$ & $28.175 \pm 0.004$ & $23.839 \pm 0.037$ & 300 \\
\hline 2001 QF331 & o3106PD & O13BL3SH & N20140823S0309.fits & r_G0303 & $56,892.45919$ & $22.895 \pm 0.033$ & $28.263 \pm 0.004$ & $22.948 \pm 0.033$ & 300 \\
\hline
\end{tabular}

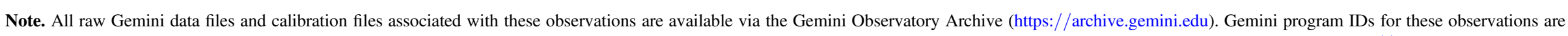

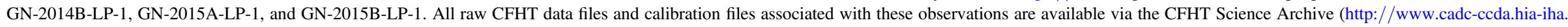

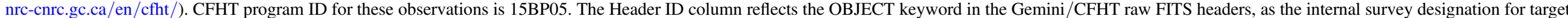

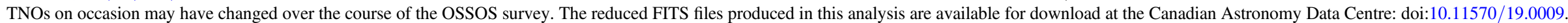

${ }^{\text {a }}$ For $J$ band, the reported exposure time is the total effective exposure time of the stacked image.

(This table is available in its entirety in machine-readable form.) 
For 2013 UX18, the standard rgJgr sequence is incomplete: the first $r_{G}$-band observation fell on a faint star and was rejected. For the 2015B observations of 2013 UN15, the CFHT data were reduced with the OSSOS procedures described in Bannister et al. (2018). Photometry was measured using TRIPPy, in the same fashion as for the Gemini data (see Appendix A). The $g-r$ color was extracted from the CFHT photometry using the line-fitting technique described in Section 4 and converted to the SDSS system using conversions provided as part of the MegaPipe pipeline (Gwyn 2008). Separately, the line-fitting technique was applied to the Gemini photometry to extract $r-z$ and $r-J$ colors in the Gemini system. Finally, the colors were converted to the SDSS system using the $g-r$ color found from the CFHT observations.

\subsubsection{Overlap with Previous Col-OSSOS Publications}

Optical colors and optical slopes derived from preliminary analysis of Col-OSSOS observations have been previously published in Fraser et al. (2017) and Pike et al. (2017) for 22 TNOs in our release sample. These targets are identified in Table 1. The same Gemini observations used in those publications are analyzed in this paper. We only report $(r-z)$ colors that were obtained during the Gemini sequences; the full $(r-z)$ sample including near-simultaneous supplemental Subaru $z$ observations can be found in Pike et al. (2017). We were able to acquire Gemini $z$ observations for five targets: 2013 SA100, 2013 SQ99, 2013 UN15, 2014 UH225, and 2014 UK225. We report the photometry and $(r-z)$ colors for those targets in Table 3 . The $(r-J)$ color values of our release sample have not been previously published, but we note that preliminary $(g-r)$ and $(J-r)$ colors for the nine TNOs that overlap with the Pike et al. (2017) sample were plotted in Figure 3 of Bannister et al. (2017a) to compare to the nearsimultaneous $g, r$, and $J$ photometry obtained for interstellar object 'Oumuamua. Marsset et al. (2019) utilizes Col-OSSOS optical colors of different OSSOS blocks that are not part of this first full data release.

The colors and photometry reported here were reprocessed using the latest version of TRIPPy (Fraser et al. 2016), the most recent data analysis pipeline. We note that small differences in the optical colors and slopes of targets reported in Fraser et al. (2017), Pike et al. (2017), and this work are due to updates to TRIPPY, improved light-curve fitting, and improved estimates of the color transformations from the Gemini filters to the SDSS photometric system, with the inclusion of additional GMOS observations. Also, during the image reductions of the 2015B Gemini observations of 2013 UN15 that were published in Pike et al. (2017), the source was contaminated by a background source in the second half of the GMOS sequence. Upon re-reduction for this work, the last image was found to be usable with a sufficiently small $r=0$. ! 8 aperture, which avoided the background star. This has caused a small adjustment in the color and a large improvement in overall color accuracy, which is reflected in the values reported in Tables 1 and 3 .

\subsubsection{Light-curve Effects}

The range of brightness variations we observed across a ColOSSOS sequence (between approximately 1 and $6 \mathrm{hr}$ duration) was $0-0.5$ mag. The photometric variability of our first-release sample is presented in Appendix $\mathrm{C}$ and the supplemental material. As described in Section 4, a linear fit was used to remove light-curve effects from our color estimates. We checked for violations of our linear model assumptions that may impact the color measurements presented here. In all cases linearity was sufficient to describe the variability we observed. By comparing the color inferred from the first half and last half of the full $r g J g r$ sequences, we found that no objects exhibited detectable spectral differences over the span of our observations. That is, the $(r-J)$ and $(g-r)$ colors that were inferred from the first half and last half of each sequence were consistent at better than $2 \sigma$ in all cases. There still may be other brightness variations present (sinusoidal being one possibility of many) that we cannot determine from our photometry and that have not been accounted for in our analysis. Such variations within our observations are unlikely to be correlated with the surface properties of the object in such a way that it then creates, artificially, the correlations between orbit and surface properties that we report in Section 6.

\section{Results and Discussion}

The $g-r$ and $r-J$ colors of the 35 TNOs in our sample are presented in Figure 4 and are reported in Table 1. There are some notable features of the optical-NIR color distribution that we discuss in this section, along with a discussion of clear outliers to the majority of the sample. Additionally, we present an analysis of the intrinsic population of objects that belong to the neutral and red classes of dynamically excited Kuiper Belt populations.

\subsection{Colors of the First-release Sample}

We present the optical and NIR color distribution of ColOSSOS targets in the 13BL and 14BH OSSOS blocks in Figure 4. We include the so-called reddening line, or line of constant spectral slope through the $g r J$ spectral range. This line was calculated using the pysynphot software package (Lim et al. 2015), using the known bandpass measurements for the SDSS $g$ and $r$ filters and the Maunakea $J$ filter. We note that the available $J$ bandpass data were measured in the laboratory at room-temperature conditions, rather than at the temperature experienced inside the NIRI dewar. ${ }^{20}$ This may cause a small deviation of the estimated $J$-band throughput away from the measured curve, and hence the calculated reddening line away from the true curve.

Substructure is apparent in the optical-NIR color space of our 35 TNOs (Figure 4). The most notable feature is the bifurcation of the dynamically excited populations into two separate color classes, as seen previously (Section 2.3). In our sample, the bifurcation into red and neutral clumps occurs at $g-r \sim 0.75$. To test for the presence of the bimodality in the Col-OSSOS observations, we apply a multidimensional test, the $F$ optimal plane (FOP) test developed in Fraser \& Brown (2012). This test uses minimal spanning tree clustering in Euclidean color space to test for the significance of potential subpopulations within a data set. We apply the FOP test to our $(g-r)$ and $(r-J)$ observations. The FOP test divides the population into two separate classes, which can be approximately divided in just the optical color, that is, at $(g-r)=0.75$, with only a $2 \%$ chance that such a division

\footnotetext{
${ }^{20}$ http://www.gemini.edu/sciops/instruments/niri/imaging/filters
} 


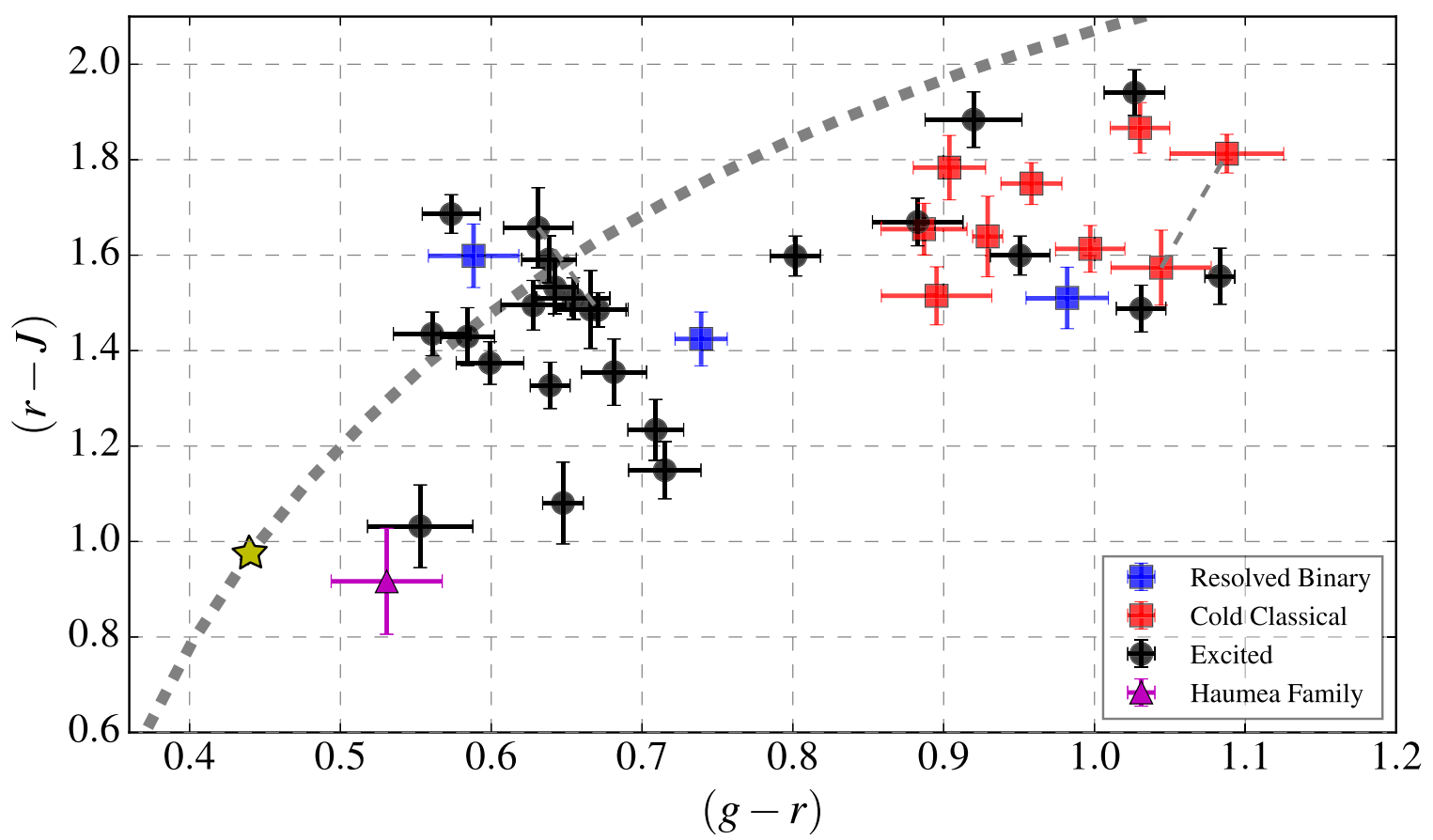

Figure 4. Optical and NIR colors of the 35 TNOs in the Col-OSSOS first-release sample (all $m_{r}<23.6$ TNOs in the OSSOS 13BL and 14BH survey blocks). The dashed curve indicates the reddening line; see Section 6.1 for details. Three objects, 2013 SQ99, 2014 UD225, and 2016 BP81 (blue squares), have cold classical orbits (main Kuiper Belt with $i<5^{\circ}$; red squares) and have been previously identified as widely separated binaries. The object 2013 UQ15 (magenta triangle) is dynamically consistent with the Haumea family. Excited TNOs (black circles) belong to the Centaur, scattering, resonant, and hot classical dynamical populations. The two measurements of the two reobserved targets, neutral hot classical 2013 SA100 and red cold classical 2013 UN15, are linked by dashed gray lines (the color measurements at each of the two observation epochs are reported in Table 1). The solar color, with $g-r=0.45$ and $r-J=0.97$, is shown by the yellow star.

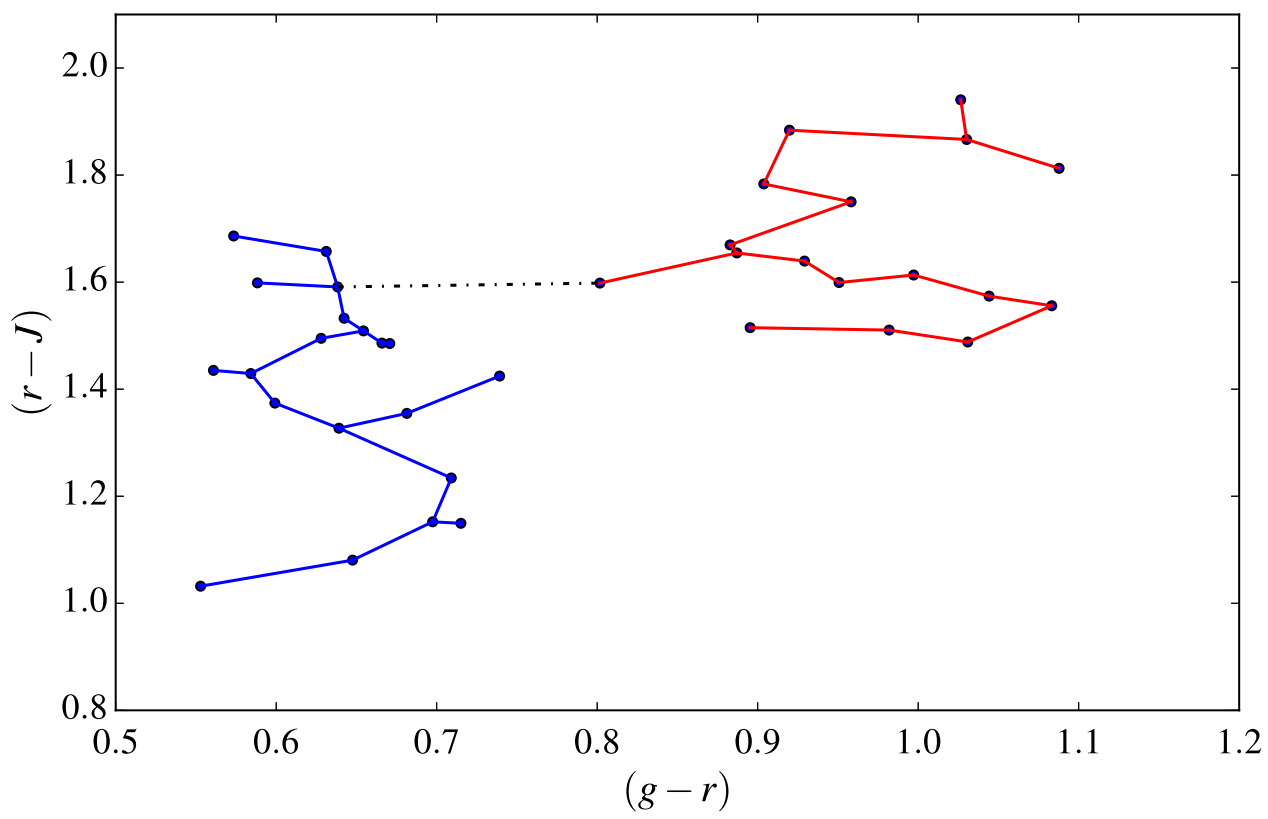

Figure 5. Minimal spanning tree, and subtrees determined by the FOP test applied to the optical and NIR colors as shown in Figure 4. The branches connecting members of the two subclasses identified by the test are shown in blue and red lines, respectively. The one branch of the full tree that is cut to result in the two subtrees is shown by the dashed black line.

would occur by chance. We present the minimum spanning tree generated by the FOP test in Figure 5.

Our sample has two objects that stand as clear outliers from the broad trend of TNO $r-J$ colors being redder than solar: 2014 UL225 and 2013 UQ15, both of which have $g-r \sim 0.55$ and $r-J \sim 0.95$ (Figure 4). 2013 UQ15 is a hot classical object with orbital elements that place it well within the cloud of objects belonging to the Haumea collisional family (Brown et al. 2007; Ragozzine \& Brown 2007). The slightly red optical color and neutral NIR color of 2014 UL225 are very similar to those exhibited by known Haumea family members, including 2005 RR43 and 1995 SM55 (Snodgrass et al. 2010), but the deep water ice absorption signature that is characteristic of Haumea family members cannot be identified with the ColOSSOS observations alone. While 2014 UL225 exhibits similar spectral properties to $2013 \mathrm{UQ} 15$, its orbital inclination of 7.9 is 
significantly lower than the Haumea cloud. We consider whether resonant diffusion, as experienced by Haumea itself (Ragozzine \& Brown 2007), could move 2014 UL225 so far away from the orbital phase space occupied by the majority of known family members. Resonant diffusion is most effective in changing the eccentricity of an orbit. 2014 UL225's eccentricity is consistent with having been affected by diffusion, but its low orbital inclination makes resonant diffusion unlikely. Volk \& Malhotra (2012) examine the long-term orbital evolution of hypothetical Haumea family members, and there are no instances of resonant diffusion down to inclinations less than $20^{\circ}$ for stable orbits. 2014 UL225's colors make it an outlier compared to the bulk of the neutral class. Further study is warranted to determine whether this object has a water-rich surface and whether it is also consistent with the Haumea family.

We find that the optical/NIR colors vary for the two TNOs (2013 SA100 and 2013 UN15) that we observed twice. We report the objects' two sets of color measurements in Figure 4 and Table 1. Spectral variability at this level has been observed in other small TNOs (see Fraser et al. 2015). We note that in neither case do the variations in colors between epochs shift the TNOs from their color class: they are consistent in color class despite the variability. For the hot classical 2013 SA100, the colors measured at each epoch both place the TNO firmly within the neutral class, but the colors are discrepant at the $1 \sigma$ level for each of the repeated $g-r, r-J$, and $r-z$ colors. Both measurements of cold classical 2013 UN15 place it in the red cloud, but the optical and NIR colors are not consistent within the $1 \sigma$ measurement uncertainty. We find that 2013 UN15 varies by $0.2 \mathrm{mag}$ or more in $r-J$ and $r-z$ (also noted by Pike et al. 2017).

The bulk of the objects with $g-r<0.75$ (the neutral class) appear to exhibit an inverse correlation in their optical and NIR colors (see Figure 4). We apply the Spearman rank test to examine this further. When we exclude the candidate Haumea collisional fragment 2013 UQ15, as it is a surface type produced via collision and not intrinsic to the planetesimal disk, the Spearman rank test suggests that there is a $30 \%$ chance that the observed correlation would occur by chance, finding no evidence that the correlation is statistically significant. The correlation becomes much stronger if we exclude 2013 UQ15 and 2014 UL225, both outliers from the excited TNO distribution. Excluding 2013 UQ15 and 2014 UL225, the Spearman rank test suggests that there is only a $2 \%$ chance that the observed correlation would occur by chance, but it is not clear that 2014 UL225 surface colors are due to some process similar to the Haumea family formation that would justify excluding it from the analysis. Correlations in the optical and NIR colors of dynamically excited TNOs have been identified previously. In particular, the NIR colors of both the neutral and red class members correlate positively with their optical colors in the Hubble Space Telescope (HST) Wide-Field Camera 3 broadband filters centered at $\sim 0.6 \mu \mathrm{m}$ (F606w), $\sim 0.8 \mu \mathrm{m} \quad(\mathrm{F} 814 \mathrm{w})$, and $\sim 1.39 \mu \mathrm{m} \quad(\mathrm{F} 139 \mathrm{~m})$ (Fraser \& Brown 2012). That result is in stark contrast to the potential inverse optical-NIR color correlation found here in the $g, r$, and $J$ filters, with band centers at $0.48,0.62$, and $1.25 \mu \mathrm{m}$, respectively. For the dynamically excited objects in the red class, optical and NIR colors exhibit a positive correlation in the HST filter set. Yet strangely, no correlation of any kind is present in the Col-OSSOS grJ observations of seven of the dynamically excited red objects in our sample (as shown in
Figure 4). This may suggest that the $J$ band samples a different part of TNO surface reflectance than the F139m HST band, but we cannot demonstrate that the correlation at the level seen in the HST band can be excluded by this sample of TNOs. A large grJ sample is required; this topic will be further investigated in future Col-OSSOS data releases.

The color trends observed in the HST filters have been used to provide compositional constraints for the bulk ice and silicate components of small TNOs (Fraser \& Brown 2012). For example, organic materials have been suggested to account for the positively correlated optical and NIR colors through a simple compositional mix: higher organic content leads to redder optical and NIR colors. If the inverse correlation that we have detected for the neutral class of dynamically excited TNOs is confirmed, then the material that is responsible for the optical-NIR color correlations of the neutral class must exhibit an absorption feature that overlaps $J$, and not the HST F139m filter, so as to account for the signs of the correlations seen in the two different filter sets. Clearly, confirmation of the inverse color correlation in the neutral class is important.

To date, no correlation between the optical and NIR colors of the cold classical objects has been detected. Rather, the bulk of cold classical TNOs exhibits a range of red optical and NIR colors, broadly spanning nearly the full range of colors exhibited by the red dynamically excited objects, though in an uncorrelated fashion. The only objects for which this does not appear to be true are the blue binaries (Fraser et al. 2017). All members of this recently discovered class of cold classical TNOs exhibit colors compatible with the neutral dynamically excited class throughout the $g r z J$ wavelength range (Pike et al. 2017 ) and exhibit a nearly $100 \%$ binary fraction (Fraser et al. 2017). If these objects are indeed survivors of a soft push-out via mean motion resonance sweep-up during the smooth phases of Neptune's outward migration (Fraser et al. 2017), then it follows that the binary cold classical objects should also exhibit a bimodal optical color distribution, like the dynamically excited TNOs. As of yet, insufficient data are available to test this assertion.

\subsection{The Color Fraction of Red/Neutral Surfaces in the Dynamically Excited Kuiper Belt}

To estimate the intrinsic fraction of objects in the neutral and red classes of dynamically excited TNOs, we consider an analytic derivation of the number of objects observed within a given OSSOS survey block. To derive that number, we consider a Kuiper Belt composed of bodies that can be described by object radius $R$, heliocentric distance $r$, and albedo $a$. Distributions in those parameters within the Kuiper Belt are thus given by $f(R), g(r)$, and $h(a)$, respectively. Here $g(r)$ will largely depend on the distribution of resonant TNOs, which are preferentially found at certain longitudes with respect to Neptune (see, e.g., Gladman et al. 2012). For resonators and other excited TNOs, no correlations between optical colors and perihelion/argument of perihelion/longitude of ascending node have been observed (Peixinho et al. 2015). Therefore, the explicit longitudinal and latitudinal structure will only affect the absolute number of observed objects at a given sky location, and not the fraction of objects in a color class at that pointing. Thus, for clarity of our derivation, we avoid writing $g$ as a function of latitude and longitude. 
The number of objects with albedos between $a$ and $a+d a$, radii $R$ and $R+d R$, and distances $r$ and $r+d r$ is given by

$$
n(R, r, a)=A f(R) g(r) h(a) d a d r d R,
$$

where $A$ is a convenience constant to determine the desired density unit; we adopt objects per square degree.

The magnitude of an object is $m=K-2.5 \log (a)+$ $5 \log (r \Delta)-5 \log (R)$, where $K$ is a constant related to the solar luminosity, $\Delta$ is the geocentric distance to the object and is a function of $r$, and we have ignored phase effects. Writing $R$ in terms of $m$ and its derivative with respect to $m$, we have

$$
\begin{aligned}
R & =\frac{r \Delta}{\sqrt{a}} 10^{\frac{K-m}{5}}, \\
d R & =\frac{-\ln 10}{5} R d m .
\end{aligned}
$$

The majority of Col-OSSOS targets have $r$-band absolute magnitudes brighter than $H_{r} \sim 8$, the approximate magnitude at which absolute magnitude distribution of the dynamically excited objects transitions from a steep power law to a shallower slope (Bernstein et al. 2004; Fuentes \& Holman 2008; Fraser \& Kavelaars 2009; Adams et al. 2014; Fraser et al. 2014). As such, we will consider only objects with $H_{r}<8$ and approximate that the size distribution is a power law of the form $f(R)=C R^{-q}$, where $C$ is a normalization constant and $q$ is the power-law slope. Substituting Equation (3) by this size distribution into Equation (1), $n$ can be defined as

$$
n(R, r, a)=\frac{\ln 10 C A}{5} R^{1-q} g(r) h(a) d a d r d m .
$$

Assuming that the Kuiper Belt is bounded by distances $r_{0} \leqslant r \leqslant r_{1}$, and objects in it have albedos with values $a_{0} \leqslant a \leqslant a_{1}$, the number of objects between magnitudes $m_{0}$ and $m_{1}$ is shown by

$$
\begin{aligned}
N= & \frac{\ln 10 C A}{5} \int_{a_{o}}^{a_{1}} \int_{r_{o}}^{r_{1}} \int_{m_{o}}^{m_{1}} h(a) a^{\left(\frac{q-1}{2}\right)} g(r) r^{1-q} \\
& \times \Delta^{1-q} 10^{\frac{(1-q)(K-m)}{5}} d m d r d a .
\end{aligned}
$$

If we substitute $q=5 \alpha+1$, where $\alpha$ is the logarithmic slope of the power law, we find

$$
\begin{aligned}
N= & \frac{\ln 10 C A}{5} \int_{a_{o}}^{a_{1}} \int_{r_{o}}^{r_{1}} \int_{m_{o}}^{m_{1}} h(a) a^{\frac{5 \alpha}{2}} g(r) r^{-5 \alpha} \\
& \times \Delta^{-5 \alpha} 10^{\alpha(m-K)} d m d r d a \\
= & \frac{C A 10^{-\alpha K}}{5 \alpha} \int_{a_{o}}^{a_{1}} \int_{r_{o}}^{r_{1}} h(a) a^{\frac{5 \alpha}{2}} g(r) r^{-5 \alpha} \\
& \times \Delta^{-5 \alpha} d r d a\left[10^{\alpha m_{1}}-10^{\alpha m_{o}}\right] .
\end{aligned}
$$

We consider a simple survey in which $m_{2} \gg m_{1}$ and has a constant efficiency $\eta$ that goes to zero at magnitude $m$. Then, the number of observed objects is given by Equation (7), and we have arrived at the general form of the cumulative luminosity function $N(<m)=10^{\alpha\left(m-m_{o}\right)}$, where $\alpha \sim 0.7$ (Fraser et al. 2008; Fuentes \& Holman 2008; Petit et al. 2011):

$$
\begin{aligned}
N(<m)= & \frac{C A 10^{-\alpha K}}{5 \alpha} \int_{a_{o}}^{a_{1}} h(a) a^{\frac{5 \alpha}{2}} d a \\
& \times \int_{r_{o}}^{r_{1}} g(r) r^{-5 \alpha} \Delta^{-5 \alpha} d r 10^{\alpha m} .
\end{aligned}
$$

Now consider that the Kuiper Belt exhibits two main color populations: the red and neutral objects. These populations differ in their albedo distributions (Stansberry et al. 2008; Fraser et al. 2014; Lacerda et al. 2014) and overall number density. If, within a given survey pointing, we assume that they share the same size and radial distributions, we can derive the observed red:neutral population ratio for a given intrinsic ratio. While the latter assumption has not been tested, no detectable size distribution differences, other than absolute number, have been detected over the observable range of the dynamically excited neutral and red classes in surveys that are sensitive to those differences (e.g., Wong \& Brown 2017). With these assumptions, if the intrinsic ratio of objects in the red and neutral populations is given by $A_{\mathrm{n}}=\gamma \mathrm{A}_{\mathrm{r}}$, then the observed ratio of the red and neutral populations, $\left.R_{\mathrm{r}, \mathrm{n}}(<m)\right)$, is

$$
R_{\mathrm{r}, \mathrm{n}}(<m)=\frac{1}{\gamma} \frac{\int_{a_{o}}^{a_{1}} h_{\mathrm{r}}(a) a^{\frac{5 \alpha}{2}} d a}{\int_{a_{o}}^{a_{1}} h_{\mathrm{n}}(a) a^{\frac{5 \alpha}{2}} d a} .
$$

For our observations, the limiting magnitude $m$ is $m_{r}=23.6$, though we note that there is no explicit dependence of Equation (8) on $m$.

While it is certainly true that the red and neutral populations exhibit a range of albedos, the true distribution is currently unknown. Thus, for simplicity and a basic first estimate of the intrinsic red:neutral fraction, we model the two populations as having a single unique albedo, $a_{\mathrm{r}}$ and $a_{\mathrm{n}}$. That is, $h_{\mathrm{r}}(\mathrm{a})=\delta\left(\mathrm{a}_{\mathrm{r}}-a\right)$ and $h_{\mathrm{n}}(\mathrm{a})=\delta\left(\mathrm{a}_{\mathrm{n}}-a\right)$, where $\delta$ is the Dirac delta function. Then we are presented with the simple red: neutral fraction relation

$$
R_{\mathrm{r}, \mathrm{n}}(<m)=\frac{1}{\gamma} \frac{a_{\mathrm{r}}^{\frac{5 \alpha}{2}}}{a_{\mathrm{n}}^{\frac{5 \alpha}{2}}},
$$

which, importantly, is independent of limiting magnitude. The mean albedos for the red and neutral populations are $a_{\mathrm{r}}=12 \%$ and $a_{\mathrm{n}}=6 \%$, respectively (Fraser et al. 2014; Lacerda et al. 2014). Thus, we find $R_{\mathrm{r}, \mathrm{n}} \sim \frac{3.4}{\gamma}$, where $\gamma$ is the intrinsic ratio of neutral to red objects in the dynamically hot TNO population.

As we are considering only the bulk of the dynamically excited TNOs, we exclude the potential Haumea family member 2013 UQ15 owing to its unique surface properties attributed to its collisional origin. We avoid counting the three targets (2014 UJ225, 2014 UQ229, and 2013 UR15) that have $H_{r}>8$ and therefore avoid the region where a single power-law size distribution is not satisfied (Bernstein et al. 2004; Fuentes \& Holman 2008; Fraser \& Kavelaars 2009; Fraser et al. 2014). Thus, in the remaining bulk sample of dynamically excited objects, there are nine neutral and three red class objects in 14BH block, and four neutral and three red objects in 13BL block. The red:neutral ratios of each block are consistent at the $2 \sigma$ level. Together, the observed ratio is $R_{\mathrm{r}, \mathrm{n}}=6 / 13$. Considering the $1 \sigma$ range on the observed ratio and Equation (9), we find $\gamma=7.4_{-3}^{+3.6}$. Thus, the observed population implies that in the intrinsic population the neutral class outnumbers the red class, by a factor of 4.4-11.0. We further note that adoption of a distribution of albedos for each class has a tendency to increase this factor substantially. For example, if we adopt uniform albedo distributions that span the observed range of albedos of the neutral and red classes $(0.04 \leqslant a \leqslant 0.08$ and $0.08 \leqslant a \leqslant 0.22)$, the inferred intrinsic ratio would be a factor of $\sim 3$ higher than what we infer using the mean albedos of each class. Thus, our result should be interpreted as a lower limit. 
Wong \& Brown (2017) adopt a different approach to determining the relative neutral:red population fraction. We note that the two color categories in Wong \& Brown (2017), "red" and "very red," are similar to our "neutral" and "red" color categories, respectively. Instead of integrating to a certain limiting magnitude, Wong \& Brown (2017) consider only objects detected in their survey to a given size, with appropriate assumptions on albedo of each of the red and neutral classes. Over a similar size range to that discussed here, they find that the intrinsic neutral-to-red number ratio is $\gamma=3.6 \pm 1.2$, where the uncertainty on this number is derived from the $1 \sigma$ Poisson range on the observed number of objects. This number is in $2 \sigma$ agreement with our measured value.

\subsection{The Structure of the Protoplanetesimal Disk}

If the separate classes of TNOs reflect the compositional structure of the protoplanetesimal disk from which they originated (see, e.g., Fraser \& Brown 2012), the presence of only two classes of dynamically excited TNO argues for a moderately compositionally homogenous disk, up to the level of our measurement uncertainty. To explain our observed optical-NIR color distribution of excited TNOs, only one compositional division would be needed, between $\sim 20$ and $\sim 30 \mathrm{au}$, where the majority of dynamically excited TNOs originated (e.g., Gomes et al. 2005a; Levison et al. 2008; Brasser \& Morbidelli 2013; Nesvorný 2015b; Nesvorný \& Vokrouhlický 2016). We note that we cannot rule out further finer color-composition structure below our measurement precision $(\sim 0.04 \mathrm{mag})$ that may exist in the dynamically excited TNO source population.

From our calculated $\gamma$, we can estimate where the division between the neutral and red classes occurred. To that end, we assume a simple disk, with a surface density described as $\Sigma(r) \propto r^{-\beta}$ with inner and outer extents of $r_{\min }$ and $r_{\max }$. To gauge the radial extent, we turn to models of the solar system's large-scale dynamical restructuring (Section 2.2). The currently favored scenario for giant planet migration and dispersal of the planetesimal disk is that of Nesvorný (2015a), in which Neptune originates at 22 au, implying a disk inner edge of $r_{\min } \sim 23 \mathrm{au}$. Objects in the dynamically excited populations originate inside the final location of the 3:2 mean motion resonance with Neptune, or $\sim 39$ au. We adopt that value for the outer edge of the disk from which dynamically excited objects originated. In the disk, we hypothesize a sharp transition distance, $r_{\mathrm{s}}$, for the original locations of the neutral and red populations. We note that our disk model is based on a simple assumption about the radial surface density distribution that at some level is not correct. For example, it is generally accepted that a sharp density gradient at $\sim 30$ au is required to halt Neptune during its late stages of migration (see, e.g., Gomes et al. 2004). Such a gradient is likely steeper than reflected in our simple model. As a result, the distance $r_{s}$ is likely interior to the value we estimate below.

Additional observational constraints also help inform the portrait of the protoplanetesimal disk. The dynamically quiescent cold classicals exhibit a different range of $r-z$ colors distinct from red dynamically excited TNOs even though they exhibit similar $r-J$ and $g-r$ colors (Pike et al. 2017). Thus, Pike et al. (2017) infer that the cold classicals are their own unique TNO surface type. It follows from this result that the disk had a second division beyond which the cold classicals originated. This second compositional division/boundary must have been near the current inner edge of the cold classical objects, to explain why cold classical-like surfaces are rare (or not present) in the dynamically excited populations. Fraser et al. (2017) found that the blue binary cold classicals, which have neutral colors consistent with the neutral excited TNO surfaces, are interlopers emplaced during Neptune migration. This places an additional constraint on where this cold classical surface boundary can be. Dynamical modeling by Fraser et al. (2017) finds that in order to deliver the blue binaries onto cold classical orbits during Neptune migration, neutral surfaces were present up to the inner edge of the cold classical belt. Thus, the transition to red cold classical surfaces would be expected near the start of the present-day cold classical belt, with red excited TNOs originating more inward than the neutral TNO surfaces.

Combining our results with these additional observational constraints, we can explore the red/neutral transition region for the source of the excited TNOs. Despite not knowing $\beta$, the power-law slope of the disk surface density, we can use the inferred intrinsic neutral-to-red population ratio to place some constraints on the transition distance. For $0 \leqslant \beta \leqslant 3$, our values of gamma imply $37.4 \leqslant r_{\mathrm{s}} \leqslant 38.5$ au if the neutral class originated inside $r_{\mathrm{s}}$, or $32.5 \leqslant r_{\mathrm{s}} \leqslant 33.4$ au if the neutral class originated outside $r_{\mathrm{s}}$. This estimate fails to account for any variation in efficiency of scattering from certain regions of the disk into different dynamical classes within the Kuiper Belt. It also fails to account for the currently unexplained sharp transition in surface density of the protoplanetesimal disk at $\sim 30 \mathrm{au}$, which is seemingly required to halt Neptune's migration at the correct distance. As such, this estimate should be taken only as a rough guide for the location of $r_{\mathrm{s}}$. A more thorough estimate will be made through the use of the OSSOS survey simulator (Lawler et al. 2018a), and forward-modeling migration model output, when a larger sample of Col-OSSOS photometry is complete.

Synthesizing all the observational constraints from Pike et al. (2017) and Fraser et al. (2017) with our results based on the assumptions described in Section 6.2, we find a protoplanetesimal disk with a red-blue-red structure, as shown in Figure 6. Closest to the Sun, today's dynamically excited red class originates at a point interior to the neutral class, with the division between the two at $\sim 33$ au. The dynamically excited neutral class starts interior to the cold classical objects, with a division between the two only a few au inside of the current inner edge of the cold classical region, at $\sim 40 \mathrm{au}$. The higher inclinations of the neutral dynamically excited TNOs (Marsset et al. 2019) imply that they have experienced a more agitated dynamical history than the red ones (Gomes 2003), potentially complicating this picture. A more detailed comparison between Col-OSSOS observations and output of dynamically compatible migration simulations will test the viability of this overall compositional picture. We also note that finer grJ colorcomposition structure that is not resolvable by our measurements may exist within each of these composition classes, further complicating this picture.

\section{Conclusions}

We present optical and NIR colors of 35 TNOs, found in the $13 \mathrm{BL}$ and 14BH OSSOS discovery blocks with magnitudes brighter than $m_{r}=23.6$. In $g-r$ and $r-J$, the dynamically excited TNOs exhibit two classes of objects: the neutral and red 


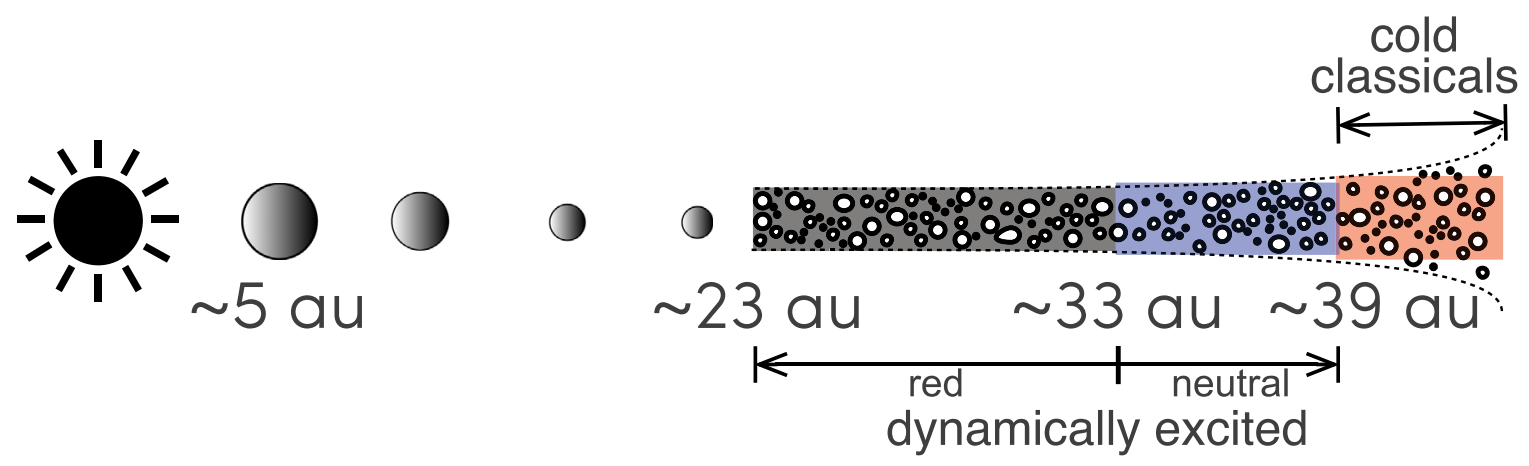

Figure 6. Implied planetesimal disk structure, not to scale, under the assumption of a moderately compositionally homogenous disk.

classes. We find a tentative negative correlation between the $g-r$ and $r-J$ colors for the neutral class, but additional observations are needed to confirm. We find no evidence for a correlation in the colors of the dynamically excited red class. Assuming a population density that is a separable function of distance, size, and albedo, we find that the neutral class outnumbers the red class by at least 4.4:1.0 but possibly as high as 11.0:1.0.

We find that the cold classical TNOs predominantly occupy the same range of colors in $g-r$ and $r-J$ as the dynamically excited, red class of TNOs. As shown by Pike et al. (2017), however, the cold classical TNOs occupy a different range of $r-z$, demonstrating that the cold classicals present a different surface than the equivalently optically red excited objects. Combining this observation with our data, we find that within our measurement uncertainty our observations are consistent with the bulk of TNOs: dynamically excited neutral, dynamically excited red, and cold classical. This excludes rare objects such as the Haumea family members (Brown et al. 2007), the volatile-bearing dwarf planet-sized bodies (Schaller \& Brown 2007; Brown 2008), or the silicate-rich TNO 2004 EW95 (Seccull et al. 2018).

Based on the assumptions and simple TNO model described in Section 6.1, we find that our observations are consistent with a planetesimal disk with two compositional divisions separating three separate classes of objects that occurred at roughly $33 \mathrm{au}$ and at just a few astronomical units inside $40 \mathrm{au}$, the current inner edge of the cold classical region. We note that further finer color-composition structure in the planetesimal disk in $g r J$ color space that is not resolvable with our measurement cannot be ruled out. To probe the possibility of finer structure in the TNO color/composition space will require measurement uncertainties smaller than 0.01 mag in $g, r$, and $J$. Our observations and past TNO color measurements are consistent with the three surface type models for the bulk of the TNO population. The presence of neutral class interlopers in the cold classical region suggests that the neutral objects bordered the inner primordial edge of the cold classical objects and the red TNOs began interior to the neutral class. The complete Col-OSSOS sample is expected to include 96 objects from five OSSOS blocks and will include additional $u$-band photometry from CFHT, acquired simultaneously alongside the Gemini observations. This future four-band data set will be used to generate a robust taxonomic system for TNOs that accounts for the correlated optical and NIR colors they exhibit.
The authors acknowledge the sacred nature of Maunakea and appreciate the opportunity to observe from the mountain. This work is based on observations from the Large and Long Program (GN-2014B-LP-1, GN-2015A-LP-1, and GN-2015BLP-1), at the Gemini Observatory, which is operated by the Association of Universities for Research in Astronomy, Inc., under a cooperative agreement with the NSF on behalf of the Gemini partnership: the National Science Foundation (United States), the National Research Council (Canada), CONICYT (Chile), Ministerio de Ciencia, Tecnología e Innovación Productiva (Argentina), and Ministério da Ciência, Tecnologia e Inovação (Brazil). This work is also based on observations obtained with MegaPrime/MegaCam, a joint project of CFHT and CEA/DAPNIA, at the Canada-France-Hawaii Telescope (CFHT), which is operated by the National Research Council (NRC) of Canada, the Institut National des Sciences de l'Univers of the Centre National de la Recherche Scientifique of France, and the University of Hawaii. We thank the Gemini North staff for their support of the Col-OSSOS program. In particular, we acknowledge the Gemini North queue coordinators, program contact scientists, science operations specialists, and the NIRI and GMOS instrument teams for their assistance. We also thank the CFHT staff for their support of the ColOSSOS program. The authors also thank the anonymous reviewer for the careful and constructive review that improved this manuscript.

M.E.S. was supported by Gemini Observatory and also in part by an Academia Sinica Postdoctoral Fellowship. M.T.B. appreciates support during Col-OSSOS from UK STFC grants ST/P0003094/1 and ST/L000709/1, the National Research Council of Canada, and the National Science and Engineering Research Council of Canada. N.P. acknowledges funding from the Portuguese FCT-Foundation for Science and Technology (ref: SFRH/BGCT/113686/2015). CITEUC is funded by National Funds through FCT-Foundation for Science and Technology (project: UID/ Multi/00611/2013) and FEDEREuropean Regional Development Fund through COMPETE 2020-Operational Programme Competitiveness and Internationalisation (project: POCI-01-0145-FEDER-006922). K.V. acknowledges support from NASA grants NNX15AH59G and NNX14AG93G.

This research used the facilities of the Canadian Astronomy Data Centre operated by the National Research Council of Canada with the support of the Canadian Space Agency. This work also made use of the Gemini Observatory Archive, NASA's Astrophysics Data System Bibliographic Services, the JPL HORIZONS web interface (https://ssd.jpl.nasa.gov/horizons.cgi), and data and 
services provided by the International Astronomical Union's Minor Planet Center. This research made use of Astropy, a community-developed core Python package for Astronomy (The Astropy Collaboration et al. 2013; Price-Whelan et al. 2018). PyRAF are products of the Space Telescope Science Institute, which is operated by AURA for NASA.

The Pan-STARRS1 Surveys (PS1) and the PS1 public science archive have been made possible through contributions by the Institute for Astronomy, the University of Hawaii, the PanSTARRS Project Office, the Max-Planck Society and its participating institutes, the Max Planck Institute for Astronomy, Heidelberg, and the Max Planck Institute for Extraterrestrial Physics, Garching, Johns Hopkins University, Durham University, the University of Edinburgh, the Queen's University Belfast, the Harvard-Smithsonian Center for Astrophysics, the Las Cumbres Observatory Global Telescope Network Incorporated, the National Central University of Taiwan, the Space Telescope Science Institute, the National Aeronautics and Space Administration under grant No. NNX08AR22G issued through the Planetary Science Division of the NASA Science Mission Directorate, the National Science Foundation grant No. AST-1238877, the University of Maryland, Eotvos Lorand University (ELTE), the Los Alamos National Laboratory, and the Gordon and Betty Moore Foundation.

Funding for the Sloan Digital Sky Survey IV has been provided by the Alfred P. Sloan Foundation, the U.S. Department of Energy Office of Science, and the Participating Institutions. SDSS-IV acknowledges support and resources from the Center for High-Performance Computing at the University of Utah. The SDSS website is www.sdss.org. SDSS-IV is managed by the Astrophysical Research Consortium for the Participating Institutions of the SDSS Collaboration, including the Brazilian Participation Group, the Carnegie Institution for Science, Carnegie Mellon University, the Chilean Participation Group, the French Participation Group, Harvard-Smithsonian Center for Astrophysics, Instituto de Astrofísica de Canarias, Johns Hopkins University, Kavli Institute for the Physics and Mathematics of the Universe (IPMU)/University of Tokyo, Lawrence Berkeley National Laboratory, Leibniz Institut für Astrophysik Potsdam (AIP), Max-Planck-Institut für Astronomie (MPIA Heidelberg), MaxPlanck-Institut für Astrophysik (MPA Garching), Max-PlanckInstitut für Extraterrestrische Physik (MPE), National Astronomical Observatories of China, New Mexico State University, New York University, University of Notre Dame, Observatário Nacional/MCTI, The Ohio State University, Pennsylvania State University, Shanghai Astronomical Observatory, United Kingdom Participation Group, Universidad Nacional Autónoma de México, University of Arizona, University of Colorado Boulder, University of Oxford, University of Portsmouth, University of Utah, University of Virginia, University of Washington, University of Wisconsin, Vanderbilt University, and Yale University.

Facilities: Gemini:Gillett (GMOS-N, NIRI), CFHT: MegaCam.

Software: astropy (The Astropy Collaboration et al. 2013; Price-Whelan et al. 2018), Gemini IRAF package (Gemini Observatory \& AURA 2016), IRAF (Tody 1986), L.A.Cosmic (van Dokkum 2001) in python, ${ }^{21}$ matplotlib (Hunter 2007), mp_ephem ${ }^{22}$ MegaPipe (Gwyn 2008), NumPy (Oliphant 2006),

\footnotetext{
21 Malte Tewes, 2010 http://obswww.unige.ch/ tewes/cosmics_dot_py/ cosmics.py_0.4/doc/index.html.

22 https://github.com/OSSOS/liborbfit
}

PyRAF, pysynphot software package (Lim et al. 2015), SExtractor (Bertin \& Arnouts 1996), SciPy (Jones et al. 2001), synphot, TRIPPy (Fraser et al. 2016).

\section{Appendix A \\ Optical Data Reduction, Photometry, and Calibration}

In this appendix we describe the data processing of the GMOS observations used in our analysis. We also describe the photometry and calibration of our optical measurements from the Gemini filter system to the SDSS (York et al. 2000; Padmanabhan et al. 2008) filter system.

\section{A.1. GMOS Data Reduction}

The GMOS images were reduced using the Gemini IRAF package $^{23}$ (Gemini Observatory \& AURA 2016) and packages from the Ureka Python and PyRAF environment. ${ }^{24}$ For each set of observations, we obtained master twilight flats and bias files $^{25}$ in the appropriate binning and bandpass. These master calibration files are produced by Gemini regularly using a large number of individual biases and flats; for each target, we used master calibration files from within a few weeks of the observations. The GMOS observations were adjusted for CCD amplifier gain, bias-subtracted, and then divided by the master twilight flat field. This removed the vast majority of the structure in the image. Boone et al. (2018) found that the readout of e2v CCDs may create differential offsets up to 4.5 ADU in the background count when comparing empty pixels of the CCD image to those with flux sources. The sky background of our images was much higher than 4.5 ADU; thus, the impact of this effect should be negligible on ColOSSOS photometry derived from GMOS-N e2v imagery. Each image was reviewed by eye, and those rare frames where the TNO's PSF was blended with a faint background galaxy or star were rejected from our later analysis.

\section{A.2. Optical Photometry}

Col-OSSOS targets move up to several pixels across the detector during each GMOS integration. Thus, to preserve photometric $\mathrm{S} / \mathrm{N}$ and avoid the use of unnecessarily large circular apertures, our measurements were made with TRIPPy (Trailed Image Photometry in Python), a dedicated software for photometry of linearly trailed sources (Fraser et al. 2016). Using TRIPPy, we model the image PSF with a $10 \times$ undersampled look-up table and a best-fit Moffat profile. PSFs were generated individually for each GMOS frame. Only wellisolated stars with photometric $\mathrm{S} / \mathrm{N}>200$ were used in the PSF generation. In rare frames where the stellar background was particularly sparse, the $\mathrm{S} / \mathrm{N}$ requirement was reduced (never lower than $\mathrm{S} / \mathrm{N}=80$ ) until at least three stars were available. Each star was manually inspected, and only those without faint contamination within $\sim 6$ FWHMs were used. Photometric aperture corrections were measured from the generated PSF. The TRIPPy PSFs were then convolved with a line, with its trail length and angle equal to that of the trailed TNO, to create trailed PSFs (TSFs). These TSFs were then used

\footnotetext{
23 http://www.gemini.edu/sciops/data-and-results/processing-software/ download

24 http://ssb.stsci.edu/ureka/

25 Obtained through the Gemini Science Archive (http://www.cadc-ccda.hiaiha.nrc-cnrc.gc.ca/en/gemini/) and Gemini Observatory Archive (https:// archive.gemini.edu/).
} 
2013 UR15

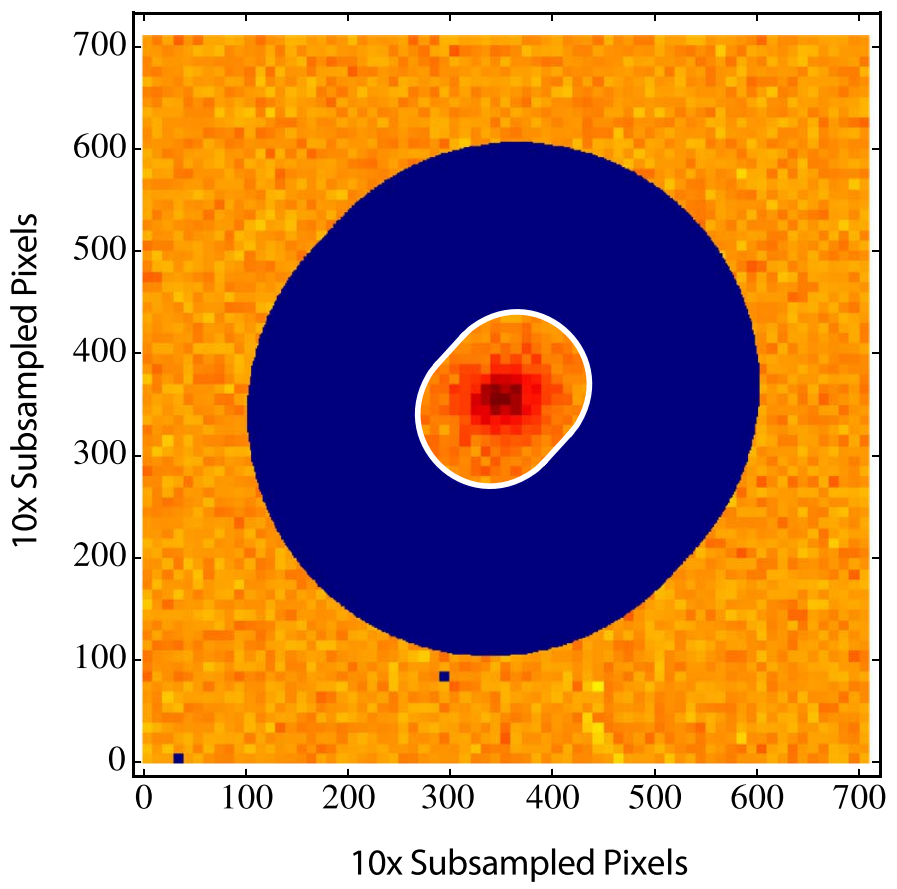

2016 BP81

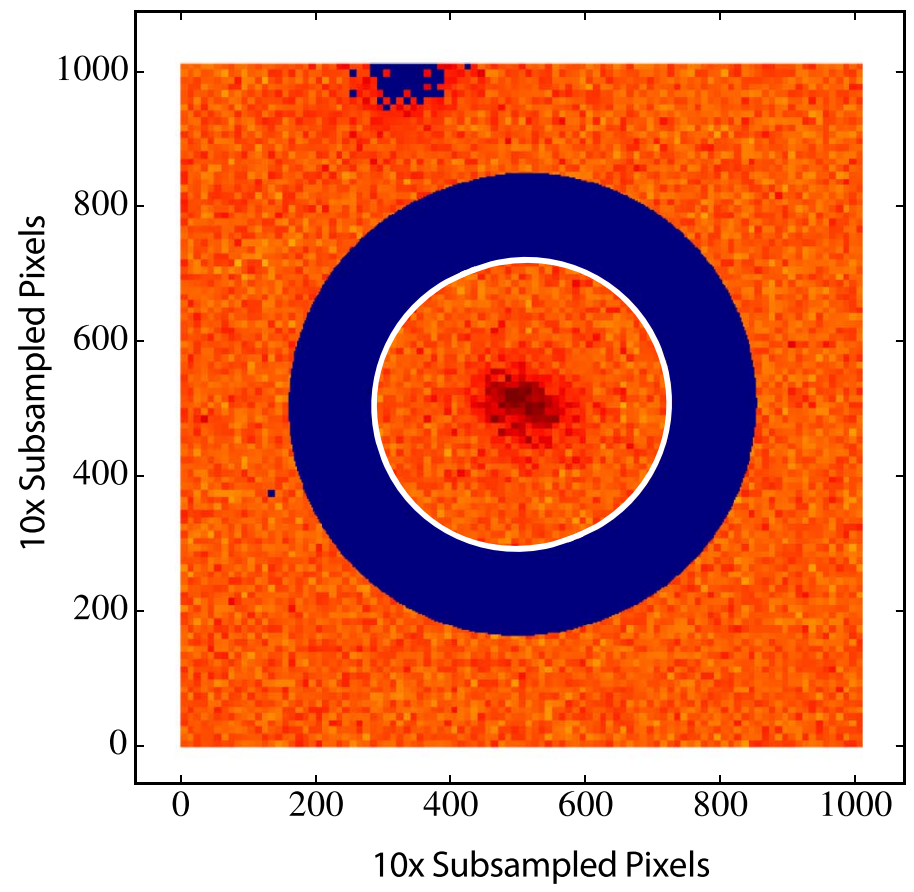

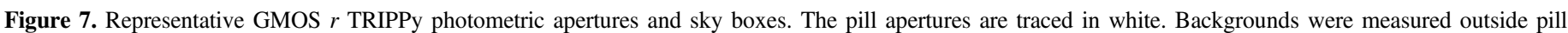

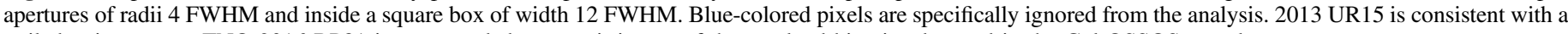
trailed point source. TNO 2016 BP81 is an extended source; it is one of the resolved binaries detected in the Col-OSSOS sample.

to compute an optimized pill-shape aperture for our target. Associated pill-shaped apertures with radii 1.2 FWHM and length equal to the trail length within the image were used to measure source fluxes. All pill fluxes were then corrected to 4 FWHM apertures using curves of growth estimated from the TSFs. Backgrounds were measured outside pill apertures of radii 4 FWHM and inside a square box of width 12 FWHM. Figure 7 shows an example TRIPPy pill aperture and sky box for representative $r$-band observations of two Col-OSSOS targets. For our reported uncertainties, we adopt the quadrature sum of the photometric shot noise, the uncertainty in color term, on-image calibration uncertainty, and the uncertainty in curve-of-growth estimate, which we take as $0.01 \mathrm{mag}$. The photometric uncertainty is dominated by the photometric shot noise, though we adopt a cautious 0.01 mag uncertainty on the aperture corrections.

An alternate choice for pill photometry would be to use PSF photometry directly. While valid if the inferred PSF and TSFs are accurate reflections of the true image shapes, this is not always the case, even with TRIPPy TSFs. As demonstrated in Fraser et al. (2016), in the TSF cores, the peak pixels in the TSF can deviate by as much as $\sim 8 \%$ compared to the true source. While a significantly smaller deviation compared to other photometry packages, this could still drive the inferred fluxes to be incorrect by a similar amount when using PSF photometry. Aperture photometry, which depends mainly on knowledge of the more correctly modeled TSF wings, is significantly less sensitive to such effects and, as such, allows more accurate flux measurements.

\section{A.3. Photometric Calibration to SDSS Photometric System}

The GMOS $g, r$, and $z$ bandpasses are similar to the filters used by SDSS (York et al. 2000); thus, we choose to report our measured colors in the more widely used SDSS Photometric
System (Fukugita et al. 1996; Padmanabhan et al. 2008). To convert from Gemini magnitudes to SDSS $\left(g_{\mathrm{S}}, r_{\mathrm{S}}, z_{\mathrm{S}}\right)$, linear color conversions between the two systems were evaluated using in-frame background stars cataloged in the SDSS (York et al. 2000) data release 13 (Albareti et al. 2017), with $g_{\mathrm{S}}<21, r_{\mathrm{S}}$ $<21$, and $0.3<\left(g_{\mathrm{S}}-r_{\mathrm{S}}\right)<1.5$ to span the full range of TNO colors, while avoiding nonlinearities in the color correction that occurs at both redder and bluer values. Our approach to measuring color terms uses a least-squares solution over all GMOS stars matched to good SDSS catalog stars in each acquired GMOS image. Our technique makes use of all ColOSSOS observations taken to date with the GMOS-N e2v CCDs (Col-OSSOS observations spanning 2014B-2016B semesters ${ }^{26}$ ). All reported Col-OSSOS optical observations taken with the $\mathrm{e} 2 \mathrm{v}$ detectors make use of our derived color terms.

Here we describe the fitting process for the Gemini $r_{\mathrm{G}}$ filter. We apply the same technique to obtain a transformation for the other filters. Circular instrumental aperture fluxes of all SDSS stars observed in GMOS frames were first acquired by TRIPPy. On image $j$, star $i$ has an $r$ magnitude in the Gemini filter system $\left(r_{i, \mathrm{G}}\right)$ as

$$
r_{i, \mathrm{G}}=Z_{j}\left(r_{\mathrm{G}}\right)-2.5 \log f_{i, j}\left(r_{\mathrm{G}}\right),
$$

where $f_{i, j}\left(r_{\mathrm{G}}\right)$ and $Z_{j}\left(r_{\mathrm{G}}\right)$ are the instrumental flux and zeropoint in the GMOS $r$ filter, respectively. The same star's magnitude can also be described by

$$
r_{i, \mathrm{G}}=r_{i, \mathrm{~S}}+C\left(g_{i, \mathrm{~S}}-r_{i, \mathrm{~S}}\right),
$$

where $r_{i, \mathrm{~S}}$ is the $r$-band magnitude for star $i$ in the SDSS filter set and $C$ is a linear color term. Equations (10) and (11) can be

\footnotetext{
26 Gemini observing programs: GN-2014B-LP-1, GN-2015A-LP-1, GN2015B-LP-1, GN-2015B-FT-26, GN-2015B-FT-28, GN-2016A-LP-1, GN2016B-LP-1.
} 
combined as

$$
r_{i, \mathrm{~S}}+2.5 \log f_{i, j}\left(r_{\mathrm{G}}\right)=Z_{j}\left(r_{\mathrm{G}}\right)-C\left(g_{i, \mathrm{~S}}-r_{i, \mathrm{~S}}\right) .
$$

For the $n$ stars on image $j$, Equation (12) can be written in matrix form as

$$
\begin{aligned}
& {\left[\begin{array}{c}
r_{1, \mathrm{~S}}+2.5 \log f_{1, j}\left(r_{\mathrm{G}}\right) \\
\vdots \\
r_{n, \mathrm{~S}}+2.5 \log f_{n, j}\left(r_{\mathrm{G}}\right)
\end{array}\right]} \\
& =\left[\begin{array}{cc}
1 & -\left(g_{1, \mathrm{~S}}-r_{1, \mathrm{~S}}\right) \\
\vdots & \vdots \\
1 & -\left(g_{n, \mathrm{~S}}-r_{n, \mathrm{~S}}\right)
\end{array}\right]\left[\begin{array}{c}
Z_{j}\left(r_{\mathrm{G}}\right) \\
C
\end{array}\right],
\end{aligned}
$$

and by extension, for all $N$ images

$$
\begin{aligned}
& {\left[\begin{array}{c}
r_{1, \mathrm{~S}}+2.5 \log f_{1,1}\left(r_{\mathrm{G}}\right) \\
r_{2, \mathrm{~S}}+2.5 \log f_{2, j}\left(r_{\mathrm{G}}\right) \\
\vdots \\
r_{n, \mathrm{~S}}+2.5 \log f_{n, N}\left(r_{\mathrm{G}}\right)
\end{array}\right]} \\
& =\left[\begin{array}{ccccc}
1 & 0 & \ldots & 0 & -\left(g_{1, \mathrm{~S}}-r_{1, \mathrm{~S}}\right) \\
0 & 1 & \ldots & 0 & -\left(g_{2, \mathrm{~S}}-r_{2, \mathrm{~S}}\right) \\
\vdots & \vdots & \ddots & \vdots & \vdots \\
0 & 0 & \ldots & 1 & -\left(g_{n, \mathrm{~S}}-r_{n, \mathrm{~S}}\right)
\end{array}\right]\left[\begin{array}{c}
Z_{1}\left(r_{\mathrm{G}}\right) \\
Z_{2}\left(r_{\mathrm{G}}\right) \\
Z_{j}\left(r_{\mathrm{G}}\right) \\
\vdots \\
Z_{N}\left(r_{\mathrm{G}}\right) \\
C
\end{array}\right]
\end{aligned}
$$

or $\boldsymbol{y}=A \boldsymbol{x}$. Solving Equation (14), $\boldsymbol{x}=\left(A^{T} \cdot A\right)^{-1} \cdot A^{T} \cdot \boldsymbol{y}$ provides the least-squares solution for all zero-points $Z_{j}\left(r_{\mathrm{G}}\right)$ and color term $C$.

Uncertainty on $C$ was found by a Monte-Carlo process. The magnitude of each star $i$ was scattered by a Gaussian variate distribution with width equal to its photometric uncertainty in the GMOS frames, and a new value $C^{\prime}$ was found. This process was repeated 200 times, with the uncertainty on $C$ taken as the standard deviation of the set of $C^{\prime}$. In total, 754 stars over 102 frames were used to determine the transformation from Gemini to the SDSS system for $r$. For $g, 753$ stars in 188 exposures were used in the least-squares fitting. $z$ had the least number of frames and sources used in the fit; 250 SDSS sources over 39 frames were employed in our $z$ color term analysis. The minimum/maximum number of stars per GMOS image used in this analysis was 3/15 for $g, 2 / 12$ for $r$, and 3/11 for $z$.

From the above analysis, the resultant color terms between the SDSS filter system and the GMOS-e2v filter system are

$$
\begin{gathered}
g_{\mathrm{G}}=g_{\mathrm{S}}-0.139( \pm 0.002) \cdot(g-r)_{\mathrm{S}}, \\
r_{\mathrm{G}}=r_{\mathrm{S}}-0.060( \pm 0.003) \cdot(g-r)_{\mathrm{S}}, \\
z_{\mathrm{G}}=z_{\mathrm{S}}-0.026( \pm 0.017) \cdot(g-r)_{\mathrm{S}} .
\end{gathered}
$$

The color terms to convert to the SDSS photometric system and the best least-squares fits are presented in Figure 8. We note that solving the inverse problem, e.g., $r_{i, \mathrm{~S}}=Z_{j}\left(r_{\mathrm{G}}\right)-$ $2.5 \log f_{i, j}\left(g_{\mathrm{G}}\right)+D\left(g_{G}-r_{G}\right)$, results in exactly the same color terms $D$ as those derived from inverting Equation (12), to within the uncertainties of the color terms $C$. We also note that for consistency between observations, and to ensure that we could convert observed $r-z$ colors into the standard SDSS system, we made use of $g-r$ in calculating the $z$ color term.
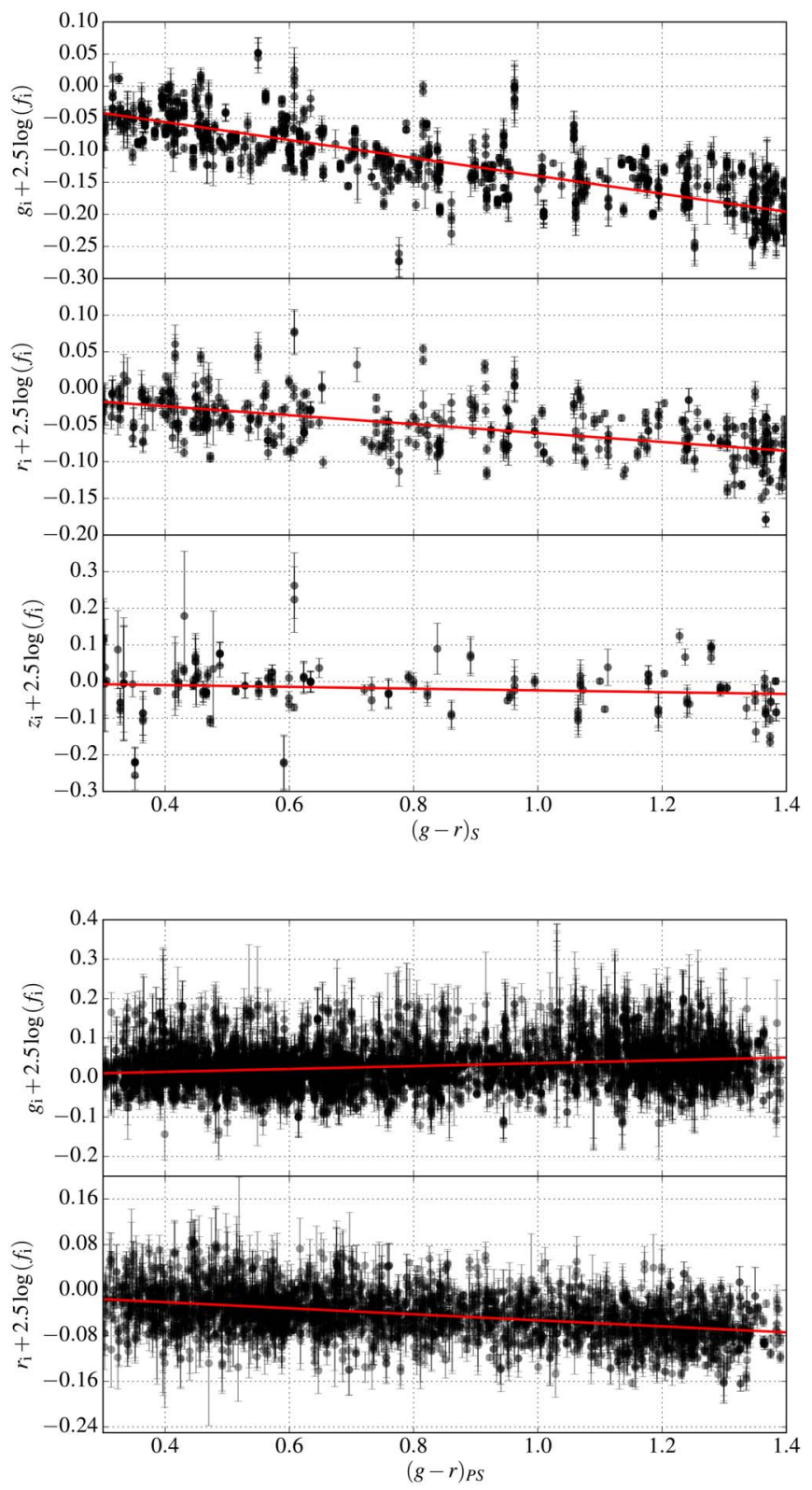

Figure 8. Difference between instrumental Gemini magnitudes and the catalog magnitudes of selected stars, vs. catalog color, for the two catalogs used for calibration in Col-OSSOS: SDSS (upper set of panels) and Pan-STARRS1 (lower set of panels). Upper set: SDSS. Top: $g$ band; middle: $r$ band; bottom: $z$ band. Lower set: Pan-STARRS1. Top: $g$ band; bottom: $r$ band. In each case, the derived best-fit linear color term is shown as a red line.

For cases where the science frames did not overlap the SDSS fields, we made use of the Pan-STARRS Data Release 1 catalog (Chambers et al. 2016; Flewelling et al. 2016; Magnier et al. 2016). The linear color conversions between the PanSTARRS and filter systems were evaluated as described above, but using only stellar-like calibration sources, chosen as those sources with Kron magnitudes and circular aperture magnitudes differing by less than $0.3 \mathrm{mag}$. The color terms as evaluated against the Pan-STARRS system are given by

$$
\begin{aligned}
& g_{\mathrm{G}}=g_{\mathrm{PS}}+0.037( \pm 0.002) \cdot(g-r)_{\mathrm{PS}} \\
& r_{\mathrm{G}}=r_{\mathrm{PS}}-0.052( \pm 0.002) \cdot(g-r)_{\mathrm{PS}} .
\end{aligned}
$$

The color terms to convert to the Pan-STARRS1 system and the best least-squares fits are presented in Figure 8. As a check 
of the precision of our color terms, we made use of our evaluated color terms to solve for a conversion of $g-r$ between the SDSS and Pan-STARRS systems directly, and we compared that to the accurate conversion between the two systems reported by Tonry et al. (2012). Through the valid range in color, $0.3 \leqslant(g-r)_{\mathrm{S}} \leqslant 1.5$, our conversion and that reported by Tonry et al. (2012) deviated by no more than 0.01 mag, demonstrating the veracity of the color terms we report.

The zero-points $\left(Z_{j}\left(r_{\mathrm{G}}\right)\right)$ (which were initially calibrated off the SDSS or Pan-STARRS magnitudes, assuming $C=0$ ) in the Gemini system are improved, by converting all cataloged magnitudes of the stars observed in a frame to the GMOS filter system using the derived color terms. The final zero-point for image $j, Z_{j}\left(r_{\mathrm{G}}\right)$, is calculated as the $3 \sigma$ clipped weighted mean of all $Z_{j, i}\left(r_{\mathrm{G}}\right)$, weighted by the inverse of the quadrature sum of SDSS/Pan-STARRS catalog uncertainty and GMOS photometric uncertainty for each star. The minimum/maximum number of stars per GMOS image used to calculate the final zero-points was $11 / 30$ for $g, 12 / 29$ for $r$, and $10 / 26$ for $z$.

\section{Appendix B \\ Near-infrared Data Reduction, Photometry, and Calibration}

In this appendix we detail the processing and analysis of the NIRI TNO and standard star observations and describe our photometric calibration of the NIRI data. After using the Gemini IRAF task nprepare (Gemini Observatory \& AURA 2016) to prepare the raw image data and add keyword information to the FITS (Flexible Image Transport System) headers, we employed custom-built python scripts to reduce the images and measure fluxes.

\section{B.1. NIRI Data Reduction}

All NIRI science and calibrator images were passed through cosmic-ray and bad pixel rejection routines. Cosmic-ray rejection was performed on each NIRI frame using the python implementation ${ }^{27}$ of the L.A.Cosmic (Laplacian Cosmic Ray Identification) algorithm (van Dokkum 2001). Bad pixel maps were created by using both the individual dark exposures and co-added nightly dark exposures to identify outlier pixels, those with unusual sensitivity behavior. Bright/hot pixels are identified from the short-exposure dark as those pixels that are 2.5 times standard deviation above the median value of the dark. Low-sensitivity pixels are those that are $4.5 \sigma$ below the median of the dark and as pixels with negative values. We then combined our bad pixel map with the one provided by the Gemini IRAF package. These flagged pixels were not used when combining the individual NIRI frames to produce the stacks. Additionally, science frames that showed a mean source flux variation of more than 50\% compared to the mean source fluxes of the entire sequence were flagged as likely suffering from extinction due to cloud or moisture and not included in the creation of the sky frames and the final image stacks.

For a given Col-OSSOS target sequence, a master sky flat was produced from the median unshifted science frames with sources masked. In order to account for temporal variations in the sky, a custom sky frame was generated uniquely for each NIRI TNO image taken. The sky frame was produced from a rolling average of the 15 temporally closest usable images with

\footnotetext{
27 https://obswww.unige.ch/ - tewes/cosmics_dot_py
}

sources masked, and avoiding common dither patterns, with the window temporally centered around the frame, whenever possible. We settled on 15 images for the sky frame after examining the frame-to-frame variation produced by differing numbers of images, as fewer frames produced a background measurement of insufficient quality from the noisy individual images. This custom sky frame was scaled to match the background level of the science frame in question and then subtracted. In our first-release sample (described in Section 5), the minimum number of NIRI frames in a TNO sequence is 7 (2010 RE188), and the maximum was 79 (2013 UN15).

The individual sky-subtracted TNO images were then mean combined in two ways: (1) a sidereal stack and (2) a nonsidereal stack. The sidereal stack is made by co-adding the individual frames using multiple star centroids in order to create a deep image of the star field and obtain point-like stars that can be used to compute the PSF. The non-sidereal stack is created by co-adding the frames shifting at the TNO's predicted on-sky velocity, as determined from propagating the OSSOS best-fit orbit to the time of observation, using the OSSOS python wrapper ${ }^{28}$ of the Bernstein \& Khushalani (2000) orbit fitter. For each TNO target, the full NIRI sequence was divided at the sequence midpoint into two half sequences, from which two sidereal and two non-sidereal stacks (called split stacks) were produced. This afforded some sensitivity to light-curve variations, which we discuss in Section 4. When the number of frames to be stacked was less than 9, a median stack was produced so as to afford some resistance to errant pixels when a small number of frames were available. Otherwise, a mean stack was produced. The minimum number of NIRI frames used in a split stack was 3 , and the maximum was 40 . The effective exposure time for each stack and the number of NIRI frames associated with each non-sidereal stack are listed in Table 3.

NIR photometric standard stars bracketed the full GMOSNIRI-GMOS TNO sequence. For each standard, the frames were aligned and median-combined to produce sidereal calibration stacks from which the standard star's flux could be measured. The standard star observations were divided by a flat field produced from the GCAL (Gemini facility calibration unit) imaging flats obtained as part of the daily calibrations, resulting in images with flat background, exhibiting brightness variations of less than $1 \%$ across the whole frame.

\section{B.2. Near-infrared Photometry and Photometric Calibration}

For the reasons discussed in Appendix A.2, we adopt pill apertures to measure photometry from our NIRI observations. The PSF was generated from the sidereal stacks. The relatively low number of nonsaturated stars, due to NIRI's small FOV, and the presence of barely resolved galaxies made it challenging to generate an accurate PSF. Particular care was therefore taken in choosing the stars used to generate the PSF. Where possible, hand-selected point-like stars with $\mathrm{S} / \mathrm{N}>100$ were used to generate the stellar PSFs and the TSF of each individual source. In a few cases where at least three satisfactory stars were unavailable, this $\mathrm{S} / \mathrm{N}$ threshold was lowered as necessary to include at least three stars in the PSF generation, to a lower limit of $\mathrm{S} / \mathrm{N}=50$. In one case, only two suitable stars were available for PSF generation, regardless of

\footnotetext{
$\overline{28}$ Available at https://github.com/OSSOS/liborbfit, and from the Python Package Index via pip install mp_ephem.
} 
2013 UR15

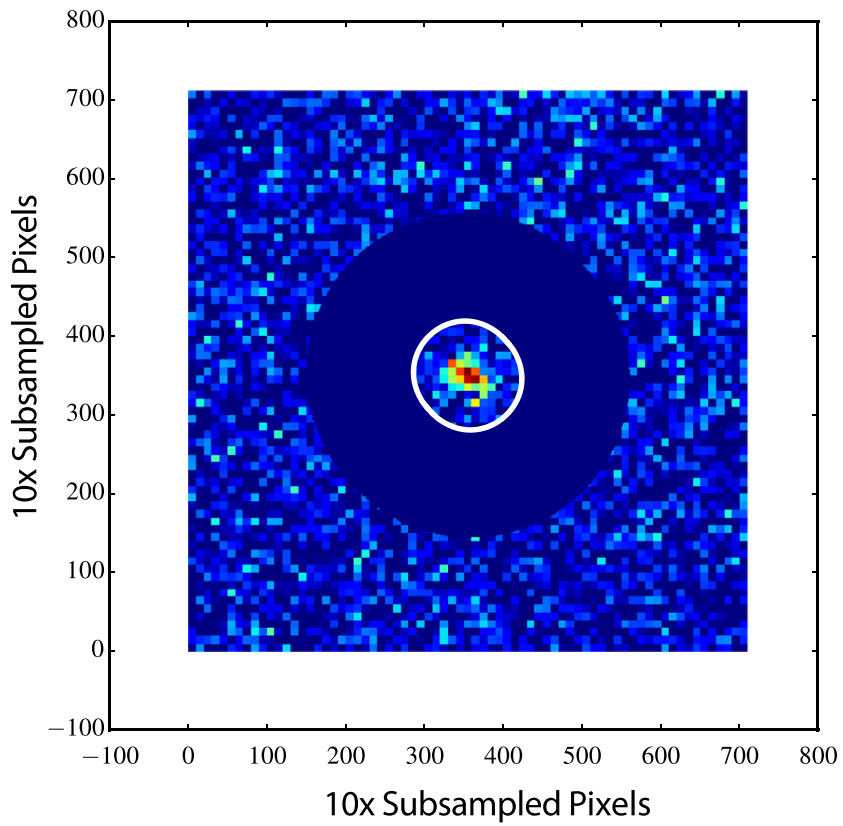

\section{BP81}

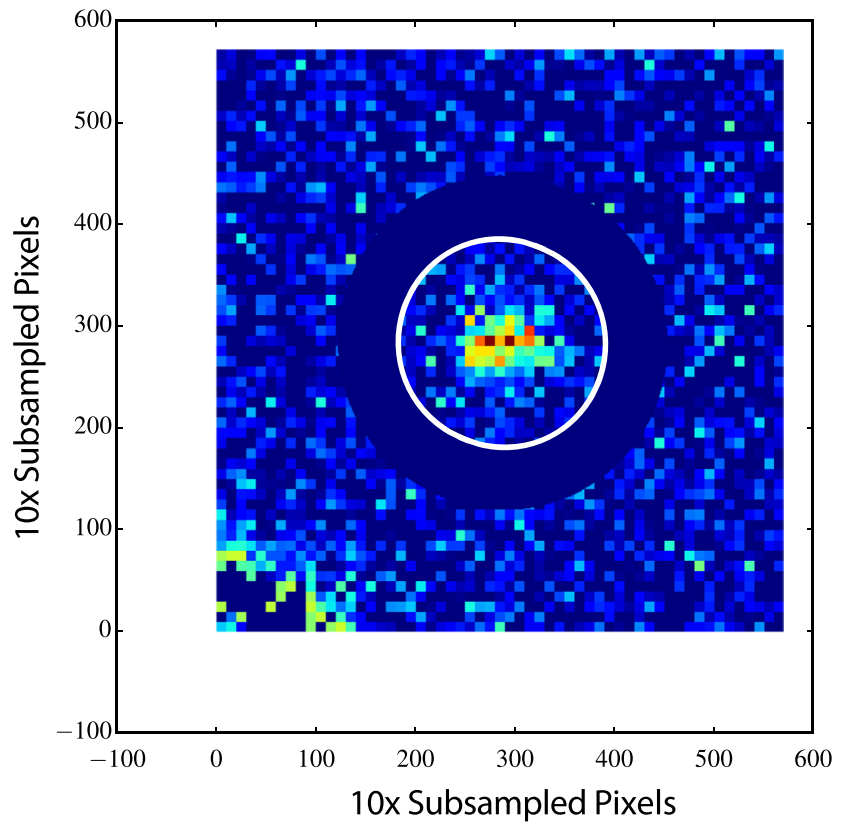

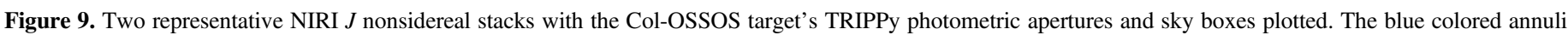

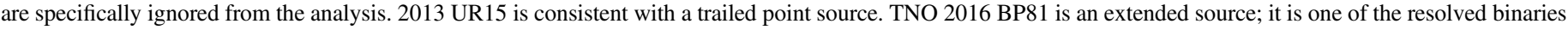
found in the Col-OSSOS sample.

the $\mathrm{S} / \mathrm{N}$ threshold. Appropriate pill apertures, tailored to each TNO target with radius 1.2 FWHM, and curve-of-growth corrections, measured from the TSF, were then used to measure the flux of the TNOs in the non-sidereal stacks. We note that the alignment necessary to produce the sidereal and non-sidereal stacks may have induced small variations in the true stacked TSF that are not properly reflected in the TSF generated by convolution of the PSF, which was generated from the sidereal stack. This would reflect in the aperture correction derived for the non-sidereal stacks. Experiments in removing the TSF profile from the science frames produced residuals of only a few percent. Thus, for our reported photometry we adopt a generous 0.02 mag uncertainty (double that of the GMOS value), which reflects this additional level of complication. Sky backgrounds were measured outside pill apertures of radii 4 FWHM and inside a square box of width 12 FWHM. Figure 9 shows the TRIPPy photometric aperture and sky box for the non-sidereal stacks of two representative Col-OSSOS targets.

We calculate each target's NIR colors based on the mean $J$ band magnitudes derived from the two split stacks. The consistency of our $J$-band photometry is demonstrated by the close match between the mean measurement derived from the two split stacks, and the measurement derived from the stack built from the full sequence of images. Specifically, for all the TNOs in our first-release data set (Section 5), both measurements agree within $0.03 \mathrm{mag}$, in agreement with our photometric precision. There is only one exception, 2010 RE188, where the two measurements agree within $0.06 \mathrm{mag}$.

The photometric uncertainty for each of the nonsidereal stacks was taken as the square root of the quadratic sum of all of the error contributions. Four main sources of uncertainty were identified in our NIR photometric measurements: the photometric shot noise, accuracy of the science stack background level measurement, zero-point measurement error, and the uncertainty due to the TSF estimation. For the background estimation error, we adopt a value of 0.02 mag for stacks with fewer than 15 images and a value of $0.01 \mathrm{mag}$ otherwise. The uncertainty on the aperture correction was calculated as the standard deviation in aperture corrections measured from each of the sources used to generate the TSF and PSF. We adopted a minimum value of $0.01 \mathrm{mag}$ if the PSF was computed from at least three stars and 0.02 for two stars. Finally, we include the 0.02 mag uncertainty as a result of the uncertain aperture correction. We find that the resultant $\mathrm{S} / \mathrm{N}$ performance is in agreement with expectations from the NIRI performance (reported by the Gemini Integration Calculator ${ }^{29}$ ) for the range of target brightnesses considered here.

Photometric calibration was preformed using the standard star observations that bracket the full NIRI-GMOS sequence. Using TRIPPy, a large 4 FWHM radius circular aperture was used to measure the flux of each calibrator and thus infer a zero-point for the calibration frame. The zero-points measured in each calibrator stack were corrected to reflect the mean airmass and precipitable water vapor reported by the Gemini weather monitors for each TNO science stack we generated. The adopted zero-point for each TNO stack was taken as the mean of the two calibration measurements acquired.

\section{Appendix C \\ Full Photometry Sequences and Photometric Variability of the OSSOS 13BL and 14BH TNO Sample}

We present in the online supplemental figures the measured photometry in each Col-OSSOS GMOS/NIRI optical-NIRoptical sequence plotted for each target TNO in the 13BL and 14BH OSSOS blocks. For each sequence, the fitted linear light curve used to derive the target's photometric colors, as described in Section 4, is also plotted. Figure 10 is presented as a representative example.

\footnotetext{
${ }^{29}$ http://www.gemini.edu/sciops/instruments/integration-time-calculators / niri-itc
} 

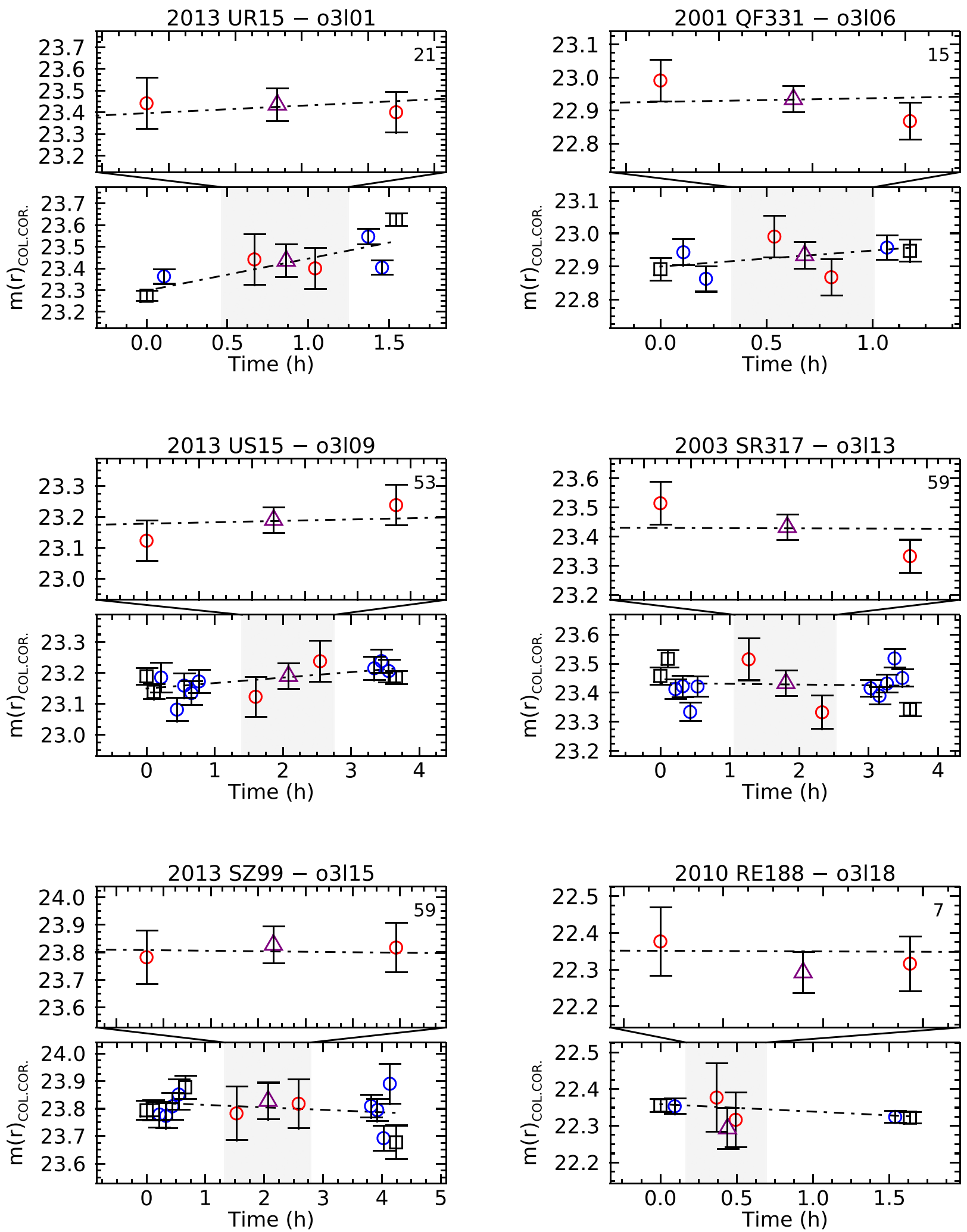

Figure 10. Col-OSSOS optical and NIR observed photometry and variability of the OSSOS 13BL and 14BH TNO sample. Each panel is divided into two subpanels showing the full GMOS/NIRI $(z) r g J g r(z)$ photometric sequence (bottom) and a zoom on the $J$-band measurements alone (top). Each measurement was color-corrected to an $r$-band magnitude using the color terms derived for the corresponding object. All optical magnitudes are in the SDSS photometric system, and $J$-band magnitudes are in the MKO system. Black squares: $r$-band magnitudes; blue circles: color-corrected $g$-band magnitudes; orange circles: color-corrected $z$-band magnitudes; red circles: color-corrected $J$-band split-stack magnitudes; purple triangles: color-corrected $J$-band full-stack magnitudes. The number of NIR frames acquired for each object is indicated in the upper right corner of each panel. The best-fit light curve for each target is shown by the dashed line. The complete figure set (6 images) for all TNOs presented in this paper is available in the online Journal.

(The complete figure set (6 images) is available.) 


\section{ORCID iDs}

Megan E. Schwamb (iD https://orcid.org/0000-00034365-1455

Wesley C. Fraser (1D https://orcid.org/0000-0001-6680-6558

Michele T. Bannister (i) https://orcid.org/0000-0003-

3257-4490

Michaël Marsset (iD https://orcid.org/0000-0001-8617-2425

Rosemary E. Pike (i) https://orcid.org/0000-0003-4797-5262

J. J. Kavelaars (i) https://orcid.org/0000-0001-7032-5255

Susan D. Benecchi (i) https://orcid.org/0000-0001-8821-5927

Matthew J. Lehner (iD https://orcid.org/0000-0003-4077-0985

Audrey Thirouin (ib https://orcid.org/0000-0002-1506-4248

Nuno Peixinho (i) https://orcid.org/0000-0002-6830-476X

Kathryn Volk (DD https://orcid.org/0000-0001-8736-236X

Mike Alexandersen (iD https://orcid.org/0000-0003-4143-8589

Ying-Tung Chen (陳英同) (10) https:// orcid.org/0000-00017244-6069

Jean-Marc Petit (iD https://orcid.org/0000-0003-0407-2266

\section{References}

Adams, E. R., Gulbis, A. A. S., Elliot, J. L., et al. 2014, AJ, 148, 55 Albareti, F. D., Allende Prieto, C., Almeida, A., et al. 2017, ApJS, 233, 25 Alexandersen, M., Benecchi, S. D., Chen, Y.-T., et al. 2018, AJ, in press (arXiv:1812.04304)

Alvarez-Candal, A., Fornasier, S., Barucci, M. A., de Bergh, C., \& Merlin, F. 2008, A\&A, 487, 741

Bannister, M. T., Gladman, B. J., Kavelaars, J. J., et al. 2018, ApJS, 236, 18 Bannister, M. T., Kavelaars, J. J., Petit, J.-M., et al. 2016, AJ, 152, 70

Bannister, M. T., Schwamb, M. E., Fraser, W. C., et al. 2017a, ApJL, 851, L38

Bannister, M. T., Shankman, C., Volk, K., et al. 2017b, AJ, 153, 262

Barkume, K. M., Brown, M. E., \& Schaller, E. L. 2008, AJ, 135, 55

Barucci, M. A., Alvarez-Candal, A., Merlin, F., et al. 2011, Icar, 214, 297

Barucci, M. A., Brown, M. E., Emery, J. P., \& Merlin, F. 2008, in The Solar System Beyond Neptune, ed. M. A. Barucci et al. (Tucson, AZ: Univ. Arizona Press), 143

Batygin, K., \& Brown, M. E. 2016, AJ, 151, 22

Batygin, K., Brown, M. E., \& Fraser, W. C. 2011, ApJ, 738, 13

Benecchi, S. D., \& Sheppard, S. S. 2013, AJ, 145, 124

Bernstein, G., \& Khushalani, B. 2000, AJ, 120, 3323

Bernstein, G. M., Trilling, D. E., Allen, R. L., et al. 2004, AJ, 128, 1364

Bertin, E., \& Arnouts, S. 1996, A\&AS, 117, 393

Boehnhardt, H., Delsanti, A., Barucci, A., et al. 2002, A\&A, 395, 297

Boone, K., Aldering, G., Copin, Y., et al. 2018, PASP, 130, 064504

Boulade, O., Charlot, X., Abbon, P., et al. 2003, Proc. SPIE, 4841, 72

Brasser, R., Duncan, M. J., \& Levison, H. F. 2006, Icar, 184, 59

Brasser, R., Duncan, M. J., Levison, H. F., Schwamb, M. E., \& Brown, M. E. 2012, Icar, 217, 1

Brasser, R., \& Morbidelli, A. 2013, Icar, 225, 40

Brasser, R., \& Schwamb, M. E. 2015, MNRAS, 446, 3788

Brown, M. E. 2001, AJ, 121, 2804

Brown, M. E. 2008, in The Solar System Beyond Neptune, ed. M. A. Barucci et al. (Tucson, AZ: Univ. Arizona Press), 335

Brown, M. E. 2012, AREPS, 40, 467

Brown, M. E., Barkume, K. M., Ragozzine, D., \& Schaller, E. L. 2007, Natur, 446, 294

Brown, M. E., Trujillo, C., \& Rabinowitz, D. 2004, ApJ, 617, 645

Brucker, M. J., Grundy, W. M., Stansberry, J. A., et al. 2009, Icar, 201, 284

Carry, B., Snodgrass, C., Lacerda, P., Hainaut, O., \& Dumas, C. 2012, A\&A, 544, A137

Chambers, K. C., Magnier, E. A., Metcalfe, N., et al. 2016, arXiv:1612.05560

Dalle Ore, C. M., Dalle Ore, L. V., Roush, T. L., et al. 2013, Icar, 222, 307

Delsanti, A., Hainaut, O., Jourdeuil, E., et al. 2004, A\&A, 417, 1145

Delsanti, A., Peixinho, N., Boehnhardt, H., et al. 2006, AJ, 131, 1851

DeMeo, F. E., Binzel, R. P., Slivan, S. M., \& Bus, S. J. 2009, Icar, 202, 160

di Sisto, R. P., Brunini, A., \& de Elía, G. C. 2010, A\&A, 519, A112

Dones, L., Gladman, B., Melosh, H. J., et al. 1999, Icar, 142, 509

Doressoundiram, A., Barucci, M. A., Romon, J., \& Veillet, C. 2001, Icar, 154,277
Doressoundiram, A., Boehnhardt, H., Tegler, S. C., \& Trujillo, C. 2008, in The Solar System Beyond Neptune, ed. M. A. Barucci et al. (Tucson, AZ: Univ. Arizona Press), 91

Doressoundiram, A., Peixinho, N., de Bergh, C., et al. 2002, AJ, 124, 2279

Doressoundiram, A., Peixinho, N., Moullet, A., et al. 2007, AJ, 134, 2186

Duffard, R., Ortiz, J. L., Thirouin, A., Santos-Sanz, P., \& Morales, N. 2009, A\&A, 505, 1283

Emel'yanenko, V. V., Asher, D. J., \& Bailey, M. E. 2003, MNRAS, 338, 443

Emery, J. P., Burr, D. M., \& Cruikshank, D. P. 2011, AJ, 141, 25

Flewelling, H. A., Magnier, E. A., Chambers, K. C., et al. 2016, arXiv:1612 05243

Fraser, W., Alexandersen, M., Schwamb, M. E., et al. 2016, AJ, 151, 158

Fraser, W. C., Bannister, M. T., Pike, R. E., et al. 2017, NatAs, 1, 0088

Fraser, W. C., \& Brown, M. E. 2012, ApJ, 749, 33

Fraser, W. C., Brown, M. E., \& Glass, F. 2015, ApJ, 804, 31

Fraser, W. C., Brown, M. E., Morbidelli, A., Parker, A., \& Batygin, K. 2014, ApJ, 782, 100

Fraser, W. C., Brown, M. E., \& Schwamb, M. E. 2010, Icar, 210, 944

Fraser, W. C., \& Kavelaars, J. J. 2009, AJ, 137, 72

Fraser, W. C., Kavelaars, J. J., Holman, M. J., et al. 2008, Icar, 195, 827

Fuentes, C. I., \& Holman, M. J. 2008, AJ, 136, 83

Fukugita, M., Ichikawa, T., Gunn, J. E., et al. 1996, AJ, 111, 1748

Gemini Observatory \& AURA 2016, Gemini IRAF: Data Reduction Software for the Gemini Telescopes, Astrophysics Source Code Library, ascl:1608.006

Gladman, B., \& Chan, C. 2006, ApJL, 643, L135

Gladman, B., Lawler, S. M., Petit, J.-M., et al. 2012, AJ, 144, 23

Gladman, B., Marsden, B. G., \& Van Laerhoven, C. 2008, in The Solar System Beyond Neptune, ed. M. A. Barucci et al. (Tucson, AZ: Univ. Arizona Press), 43

Gomes, R., Levison, H. F., Tsiganis, K., \& Morbidelli, A. 2005a, Natur, 435,466

Gomes, R., Nesvorný, D., Morbidelli, A., Deienno, R., \& Nogueira, E. 2018, Icar, 306, 319

Gomes, R. S. 2003, Icar, 161, 404

Gomes, R. S., Fern Ndez, J. A., Gallardo, T., \& Brunini, A. 2008, in The Solar System Beyond Neptune, ed. M. A. Barucci et al. (Tucson, AZ: Univ. Arizona Press), 259

Gomes, R. S., Gallardo, T., Fernández, J. A., \& Brunini, A. 2005b, CeMDA, 91, 109

Gomes, R. S., Morbidelli, A., \& Levison, H. F. 2004, Icar, 170, 492

Gulbis, A. A. S., Elliot, J. L., Adams, E. R., et al. 2010, AJ, 140, 350

Gwyn, S. D. J. 2008, PASP, 120, 212

Hainaut, O. R., Boehnhardt, H., \& Protopapa, S. 2012, A\&A, 546, A115

Hodapp, K. W., Jensen, J. B., Irwin, E. M., et al. 2003, PASP, 115, 1388

Hook, I. M., Jørgensen, I., Allington-Smith, J. R., et al. 2004, PASP, 116, 425

Horner, J., \& Lykawka, P. S. 2010, MNRAS, 402, 13

Hunter, J. D. 2007, CSE, 9, 90

Jester, S., Schneider, D. P., Richards, G. T., et al. 2005, AJ, 130, 873

Jones, E., Oliphant, T., Peterson, P., et al. 2001, SciPy: Open Source Scientific Tools for Python, http://www.scipy.org

Jones, R. L., Gladman, B., Petit, J.-M., et al. 2006, Icar, 185, 508

Jordi, K., Grebel, E. K., \& Ammon, K. 2006, A\&A, 460, 339

Kaib, N. A., \& Quinn, T. 2008, Icar, 197, 221

Kavelaars, J., Jones, L., Gladman, B., Parker, J. W., \& Petit, J.-M. 2008, in The Orbital and Spatial Distribution of the Kuiper Belt, ed. M. A. Barucci et al. (Tucson, AZ: Univ. Arizona Press), 59

Lacerda, P., Fornasier, S., Lellouch, E., et al. 2014, ApJL, 793, L2

Lawler, S. M., Kavelaars, J. J., Alexandersen, M., et al. 2018a, FrASS, 5, 14

Lawler, S. M., Pike, R. E., Kaib, N., et al. 2018b, AJ, in press (arXiv:1808. 02618)

Levison, H. F., Morbidelli, A., Van Laerhoven, C., Gomes, R., \& Tsiganis, K. 2008, Icar, 196, 258

Lim, P. L., Diaz, R. I., \& Laidler, V. 2015, PySynphot Users Guide (Baltimore, MD: STScI)

Luu, J., \& Jewitt, D. 1996, AJ, 112, 2310

Magnier, E. A., Schlafly, E. F., Finkbeiner, D. P., et al. 2016, arXiv:1612. 05242

Malhotra, R. 1995, AJ, 110, 420

Marsset, M., Fraser, W. C., Pike, R. E., et al. 2019, AJ, 157, 94

Miyazaki, S., Komiyama, Y., Sekiguchi, M., et al. 2002, PASJ, 54, 833

Morbidelli, A., \& Levison, H. F. 2004, AJ, 128, 2564

Nesvorný, D. 2015a, AJ, 150, 68

Nesvorný, D. 2015b, AJ, 150, 73

Nesvorný, D., \& Vokrouhlický, D. 2016, ApJ, 825, 94 
Noll, K. S., Grundy, W. M., Stephens, D. C., Levison, H. F., \& Kern, S. D. 2008, Icar, 194, 758

Oliphant, T. E. 2006, A Guide to NumPy

Padmanabhan, N., Schlegel, D. J., Finkbeiner, D. P., et al. 2008, ApJ, 674,1217

Parker, A. H., \& Kavelaars, J. J. 2010, ApJL, 722, L204

Peixinho, N., Boehnhardt, H., Belskaya, I., et al. 2004, Icar, 170, 153

Peixinho, N., Delsanti, A., \& Doressoundiram, A. 2015, A\&A, 577, A35

Peixinho, N., Delsanti, A., Guilbert-Lepoutre, A., Gafeira, R., \& Lacerda, P. 2012, A\&A, 546, A86

Peixinho, N., Doressoundiram, A., Delsanti, A., et al. 2003, A\&A, 410, L29

Peixinho, N., Lacerda, P., \& Jewitt, D. 2008, AJ, 136, 1837

Petit, J.-M., Kavelaars, J. J., Gladman, B. J., et al. 2011, AJ, 142, 131

Petit, J.-M., Kavelaars, J. J., Gladman, B. J., et al. 2017, AJ, 153, 236

Pfalzner, S., Bhandare, A., Vincke, K., \& Lacerda, P. 2018, ApJ, 863, 45

Pike, R. E., Fraser, W. C., Schwamb, M. E., et al. 2017, AJ, 154, 101

Pike, R. E., \& Kavelaars, J. J. 2013, AJ, 146, 75

Price-Whelan, A. M., Sipócz, B. M., Günther, H. M., et al. 2018, AJ, 156, 123

Ragozzine, D., \& Brown, M. E. 2007, AJ, 134, 2160

Ribeiro de Sousa, R., Gomes, R., Morbidelli, A., \& Vieira Neto, E. 2018, arXiv: 1808.02146

Schaller, E. L., \& Brown, M. E. 2007, ApJL, 659, L61

Schaller, E. L., \& Brown, M. E. 2008, ApJL, 684, L107

Seccull, T., Fraser, W. C., Puzia, T. H., Brown, M. E., \& Schönebeck, F. 2018, ApJL, 855, L26

Sheppard, S. S., \& Trujillo, C. 2016, AJ, 152, 221
Simons, D. A., \& Tokunaga, A. 2002, PASP, 114, 169

Snodgrass, C., Carry, B., Dumas, C., \& Hainaut, O. 2010, A\&A, 511, A72

Stansberry, J., Grundy, W., Brown, M., et al. 2008, in The Solar System Beyond Neptune, ed. M. A. Barucci et al. (Tucson, AZ: Univ. Arizona Press), 161

Tancredi, G., \& Favre, S. 2008, Icar, 195, 851

Tegler, S. C., \& Romanishin, W. 2000, Natur, 407, 979

Tegler, S. C., \& Romanishin, W. 2003, Icar, 161, 181

Tegler, S. C., Romanishin, W., \& Consolmagno, G. J. 2003, ApJL, 599, L49

The Astropy Collaboration, Robitaille, T. P., Tollerud, E. J., et al. 2013, A\&A, 558, A33

Thirouin, A., \& Sheppard, S. S. 2018, AJ, 155, 248

Thommes, E. W., Duncan, M. J., \& Levison, H. F. 1999, Natur, 402, 635

Tiscareno, M. S., \& Malhotra, R. 2003, AJ, 126, 3122

Tody, D. 1986, Proc. SPIE, 627, 733

Tonry, J. L., Stubbs, C. W., Lykke, K. R., et al. 2012, ApJ, 750, 99

Trilling, D. E., \& Bernstein, G. M. 2006, AJ, 131, 1149

Trujillo, C. A., \& Sheppard, S. S. 2014, Natur, 507, 471

Trujillo, C. A., Sheppard, S. S., \& Schaller, E. L. 2011, ApJ, 730, 105

van Dokkum, P. G. 2001, PASP, 113, 1420

Volk, K., \& Malhotra, R. 2012, Icar, 221, 106

Volk, K., Murray-Clay, R. A., Gladman, B. J., et al. 2018, AJ, 155, 260

Wong, I., \& Brown, M. E. 2016, AJ, 152, 90

Wong, I., \& Brown, M. E. 2017, AJ, 153, 145

York, D. G., Adelman, J., Anderson, J. E., Jr., et al. 2000, AJ, 120, 1579

Yu, Q., \& Tremaine, S. 1999, AJ, 118, 1873 